\title{
Computational Aeroelastic Analysis of Ares Crew Launch Vehicle Bi-Modal Loading
}

\author{
Steven J. Massey* and Pawel Chwalowski, \\ NASA Langley Research Center, Hampton, VA 23681-2199
}

\begin{abstract}
A Reynolds averaged Navier-Stokes analysis, with and without dynamic aeroelastic effects, is presented for the Ares I-X launch vehicle at transonic Mach numbers and flight Reynolds numbers for two grid resolutions and two angles of attack. The purpose of the study is to quantify the force and moment increment imparted by the sudden transition from fully separated flow around the crew module - service module junction to that of the bi-modal flow state in which only part of the flow reattaches. The bi-modal flow phenomenon is of interest to the guidance, navigation and control community because it causes a discontinuous jump in forces and moments. Computations with a rigid structure at zero zero angle of attack indicate significant increases in normal force and pitching moment. Dynamic aeroelastic computations indicate the bi-modal flow state is insensitive to vehicle flexibility due to the resulting deflections imparting only very small changes in local angle of attack. At an angle of attack of $2.5^{\circ}$, the magnitude of the pitching moment increment resulting from the bi-modal state nearly triples, while occurring at a slightly lower Mach number. Significant grid induced variations between the solutions indicate that further grid refinement is warranted.
\end{abstract}

\section{Note to Readers}

The predicted performance and certain other features and characteristics of the Ares I-X launch vehicle are defined by the U.S. Government to be Sensitive But Unclassified (SBU). Therefore, details have been removed from all plots and figures.

\section{Nomenclature}

\author{
Roman Symbols \\ a Source strength \\ C Case number \\ $C_{M X} \quad$ Coefficient of rolling moment \\ $C_{M Y} \quad$ Coefficient of pitching moment \\ $C_{M Z} \quad$ Coefficient of yawing moment \\ $C_{p} \quad$ Coefficient of pressure \\ $C_{X} \quad$ Coefficient of axial force \\ $C_{Y} \quad$ Coefficient of side force \\ $C_{Z} \quad$ Coefficient of normal force \\ $M \quad$ Mach number \\ $M_{c} \quad$ Critical Mach number \\ $q \quad$ Dynamic pressure; generalized displacement
}

${ }^{*}$ Aerospace Engineer, Aeroelasticity Branch, MS 340, Senior Member AIAA 
$|\boldsymbol{r}| \quad$ Euclidean distance between a target point and a source

$r \quad$ Radial coordinate

$r_{1} \quad$ Geometric rate of growth of boundary layer grid spacing

$r_{2} \quad$ Exponential rate of growth of boundary layer grid spacing

RE Reynolds number

$\mathrm{X}, \mathrm{Y}, \mathrm{Z}$ Body coordinate system

$y^{+} \quad$ Dimensionless, sublayer-scaled wall coordinate of first node away from surface

\section{Greek Symbols}

$\alpha \quad$ Angle of attack

$\Delta_{0} \quad$ First cell height in boundary layer

$\Delta C \quad$ Bi-modal induced load coefficient increment

$\Delta C / \Delta X$ Line load of coefficient $C$ with respect to $X$

$\Delta_{n} \quad n^{t h}$ viscous layer spacing, Eq. 1

$\Delta \bar{s} \quad$ Characteristic length (average mesh spacing)

$\Delta s_{0} \quad$ Primary grid spacing at the source

$\Delta s_{|\boldsymbol{r}|} \quad$ Primary grid spacing at the target point as a function of $|\boldsymbol{r}|$, Eqs. 2 and 3

$\phi \quad$ Azimuthal coordinate

$\theta_{c} \quad$ Cone half angle

$\Gamma_{1} \quad$ Geometric rate of growth of grid spacing from a source

$\Gamma_{2} \quad$ Exponential rate of growth of grid spacing from a source

$\eta \quad$ Hybrid growth exponent

\section{Acronyms}

CFD Computational Fluid Dynamics

CM Crew Module

IFLIM FUN3D flux limiter number

IVM12 Integrated Vehicle Model 12

LAS Launch Abort System

RCS Reaction Control System

SA Spacecraft Adapter

SM Service Module 


\section{Introduction}

Reynolds averaged Navier-Stokes analysis, including dynamic aeroelastic effects, is performed on the Ares I-X launch vehicle, Figure 1, at transonic Mach numbers and flight Reynolds numbers for two grid resolutions. Of primary interest in this study is the onset of flow reattachment around the crew module-service module junction (Figure 2) with increasing Mach number. The conical design of the Ares I-X crew module exposes the vehicle to unsteady loads due to the sudden transition from separated to attached flow about the cone-cylinder junction. The phenomenon of alternating attached and separated flow at cone-cylinder junctions was first described by Chevalier and Robertson ${ }^{1,2}$ in a detailed analysis based on measurements at several cone angles and transonic Mach numbers. The first aeroelastic analysis was performed by Ericsson. ${ }^{3,4}$ Recent literature reviews of this and other sources of fluctuating pressures on launch vehicles including the Ares I-X are given by Baker, ${ }^{5}$ Streett ${ }^{6}$ and most recently by Sekulaet al. ${ }^{7}$

The flow is considered to be bi-modal when both attached and separated flow states exist simultaneously, which can result from a non-zero angle of attack, asymmetries in the surface geometry or the unsteady transition from one state to the other. At particular Mach numbers this transition can happen repeatedly because of the high sensitivity of the flow field to the wakes of the LAS nozzles and other disturbances. An example of the bi-modal flow state is shown in Figure 3(a) together with its pressure footprint in Figure 3(b). Notice that in Figure 3(a) the cutting plane is through $\phi=45^{\circ}$ (on top) and $\phi=-135^{\circ}$ (on bottom), which bisects the separated region of high pressure as seen in the unrolled surface plot. All of the other cutting planes shown in this paper are through $\phi=0^{\circ}$ (on top) and $\phi=180^{\circ}$ (on bottom). Off surface contours are colored by Mach number with the sonic line shown as dashed. Streamlines, shown in white and confined to the surface and the cutting plane, indicate a large area of separated recirculating flow in the high pressure region, from $\phi=10^{\circ}$ to $\phi=90^{\circ}$ and as far back as the $X=1100$ in. station as shown in Figure 3(b). While on the lower half of the cutting plane corresponding to $\phi=-135^{\circ}$, the flow is seen to be attached and accelerating around the CM-SM junction creating a low pressure footprint before terminating in a shock, Figure 3(a). The adverse pressure gradient of the shock then causes flow separation, which can be seen in the Mach number contours of blue and purple just over the SA region in Figure 3(a). Note in Figure 3(b) and many other figures in this paper, a header was automatically generated in the plotting process which denotes the case number, Mach number, angle of attack, Reynolds number per $\mathrm{ft}$ in millions and the flux limiter code used. This header is also the name of the directory where the data files reside and is retained to confirm the identity of the data source.

This numerical study was originally conducted as a supplement to the Ares I-X Rigid Buffet Test, ${ }^{8}$ conducted in the Langley Transonic Dynamics Tunnel, to aid in the visualization of the flow physics and characterize the Mach number range of the bi-modal state. Later, more quantitative results were desired so computations were done on an existing finer grid to begin the verification process, the full results of which are contained in Ref. 9. Since the computations were at flight Reynolds number, no comparisons with wind tunnel data have been made.

\section{Numerical Method}

\section{A. Grid Generation}

The vehicle geometry modeled was based on the outer mold line revision AI1-SYS-OML version 2.00. Unstructured tetrahedral grids were generated using VGRID ${ }^{10}$ with input prepared using GridTool. ${ }^{11}$ The boundary layer was fully gridded with a first cell height corresponding to $y^{+} \approx 0.3$. Two previously existing grids were used in this study: a baseline grid of 22 million nodes and a fine grid of 48 million nodes. For the $n^{\text {th }}$ viscous layer spacing defined by Eq. 1, both grids used the same initial cell height of $\Delta_{0}=0.0001$ in. and geometric growth rate of $r_{1}=0.18$, while differing in the exponential growth rate, $r_{2}$, with the baseline grid having a value of 0.02 and the fine grid $r_{2}=0.025$.

$$
\Delta_{n}=\Delta_{0}\left[1+r_{1}\left(1+r_{2}\right)^{n-1}\right]^{n-1}
$$

The off surface length scale growth rate is governed by parameters $\Gamma_{1}$ and $\Gamma_{2}$ according to the following equations:

$$
\Delta s_{|r|}=\Delta s_{0}\left(1+\frac{\Gamma_{1}}{a}\right)^{\eta}
$$

where

$$
\eta=\left(1+\frac{|\boldsymbol{r}|}{\Delta \bar{s}} \Gamma_{2}\right) \frac{\ln \left(1+\frac{|\boldsymbol{r}| \Gamma_{1}}{\Delta s_{0} a}\right)}{\ln \left(1+\frac{\Gamma_{1}}{a}\right)}
$$


For the baseline grid $\Gamma_{1}$ and $\Gamma_{2}$ were both set to 0.15 , while for the fine grid the values were reduced to 0.10 and 0.12 respectively to slow the inviscid cell size growth rate. The lower value for $\Gamma_{1}$ was required to obtain grid closure. Lastly, the global inviscid refinement parameter ifact was reduced from one to 0.85 for the fine grid. Though the boundary layers are very nearly the same, these grids are not in a family of successive refinements because of the differences in the volume length scale growth rates. This lack of uniform refinement results in small features being better resolved on the fine grid, while further away the grid is actually coarser. This local coarsening on the fine grid can be seen in the off body separation region above the SM in Figure 4 and around the RCS module on the SM in Figure 5. The single most important grid effect is due to increased grid resolution at the cone-cylinder junction at the interface of the CM and SM where the flow separation begins, Figure 6.

\section{B. Rigid Steady Flow Analysis}

Solutions to the Reynolds averaged Navier-Stokes (RANS) equations are computed using the FUN3D ${ }^{12,13}$ flow solver. Turbulence closure is obtained using the Spalart-Allmaras ${ }^{14}$ one-equation model. For the Mach number range under consideration, flux limitation is not required and therefore is not used for simplicity. Inviscid fluxes are computed using the Low-Diffusion Flux-Splitting Scheme of Edwards. ${ }^{15}$ For the asymptotically steady cases under consideration, time integration is accomplished by an Euler implicit backwards difference scheme, with local time stepping to accelerate convergence. Most of the cases in this study were run for 6000 iterations to achieve convergence of forces and moments to well within $\pm 0.5 \%$ of the average of their last 1000 iterations.

\section{Dynamic Aeroelastic Analysis}

Recently, a dynamic aeroelastic capability was added to the FUN3D solver. ${ }^{16}$ For the structural dynamics, FUN3D is capable of being loosely coupled with an external finite element solver ${ }^{17}$ or in the case of linear structural dynamics used in this study, an internal modal structural solver can be utilized. The modal solver is formulated and implemented in FUN3D similarly to previous Langley aeroelastic codes, CAP-TSD ${ }^{18}$ and CFL3D. ${ }^{19}$ Structural modes were obtained via a normal modes analysis (solution 103) with the FEM solver MSC Nastran ${ }^{\mathrm{TM} 20}$ using the IVM12 finite element model of the Ares I-X launch vehicle.

The dynamic aeroelastic analysis is performed in a three-step process. First the steady CFD solution is obtained on the rigid vehicle, then a static aeroelastic solution is obtained by continuing the CFD analysis in a time accurate unsteady mode and allowing the structure to deform, but with high structural damping so the structure can find its equilibrium position with respect to the mean flow before the dynamic response is started. For the dynamic response, the damping is set to the expected value and the structure is perturbed in generalized velocity for each of modes included in the structural model and the unsteady flow is solved time accurately. Details of the FUN3D aeroelastic solution process for Ares I-X are contained in Bartels et al. ${ }^{21}$

\section{Post Processing}

Integrated forces and moments are produced directly by FUN3D. Line loads are computed using the code of Samareh. ${ }^{22}$ Unrolled surface plots are created using an external Fortran code and Tecplot ${ }^{\mathrm{TM}} 23$ to cleanly divide and transform the surface mesh into arc length versus axial coordinates. Simulated schlieren plots are created by taking the logarithm of the in-plane gradient of density and plotting in gray scale. Visualization using a logarithmic scale allows many orders of magnitude of the density gradient to be revealed in the same plot.

\section{Rigid Steady Flow Results at Zero Angle of Attack}

In the course of identifying the Mach number range of the bi-modal phenomenon, 13 baseline grid cases and 11 fine grid cases were computed at Reynolds numbers along the Ares I-X nominal ascent trajectory in Table 1 . To reduce the number of variables that could be responsible for the onset of the bi-modal flow state, the Reynolds number was held constant at 4.086 million per ft for all points bracketing the bi-modal flow state. It is very unlikely that even a linear interpolation of the trajectory would have made any difference due to the small variation of Reynolds number over the complete bi-modal Mach number range.

Results are presented in order of increasing level of output detail, starting from integrated forces and moments, Tables 2 and 3 and Figure 7, to line loads, Figures 8-13, to unrolled surface pressures, Figure 14, to Mach number and simulated schlieren flow field plots, Figures 15 and 16. 
Table 1. Ares I-X Nominal Ascent Trajectory Points. ${ }^{a}$

\begin{tabular}{rrrrr}
\hline \hline$M$ & Time, sec & Altitude, ft & $q, \mathrm{psf}$ & $R e$ per $\mathrm{ft}$ \\
\hline 0.90 & 34.9 & 14,906 & 668 & $4,086,552$ \\
0.95 & 36.7 & 16,417 & 701 & $4,129,046$ \\
1.00 & 38.4 & 18,005 & 730 & $4,150,666$ \\
\hline \hline
\end{tabular}

${ }^{a}$ Revision 4, November 9, 2007

\section{A. Integrated Forces and Moments}

Integrated forces and moments over the entire vehicle for a Mach number range of 0.88 to 0.98 on baseline and fine grids, plotted in Figure 7, indicate the solution lacks grid convergence with respect to the bi-modal phenomenon. The large relative differences in solutions between grid cases shown in Table 2, reflect two separate causes: 1) at zero angle of attack all forces normal to the vehicle and all moments are relatively small, because they are the direct result of the non-symmetric protuberances on the vehicle and 2) differences in the Mach number corresponding to the start of the bi-modal flow state. For both grid cases the axial force, $C_{X}$, is the clearest indicator of both effects where, for Mach numbers completely out of the bi-modal range, the difference is from 3-5\%. Note, the bi-modal state can be verified by examining the direction of the near surface streamlines plotted in Figures 15 and 16 of Section D.

Bi-modal flow state is characterized by a spike in integrated forces and moments. The difference or delta in loading can be seen in Figure 7 . The finer grid is seen to result in about half as much change in normal quantities such as $C_{Y}$, $C_{Z}, C_{M Y}$ and $C_{M Z}$, while resulting in increased axial loads, $C_{X}$ and $C_{M X}$. The decrease in normal loads is a result of the much smaller bi-modal state in the fine grid, Figures $14(\mathrm{~d})$ and $14(\mathrm{~g})$. The unrolled surface plots will be discussed fully in Section C. The smaller bi-modal state of the fine grid also explains the more abrupt increase in $C_{X}$, since there is a more complete removal of the negative skin friction contribution of the separated flow around the SM. The large relative difference in the rolling moment between grids is due to the values being very small for zero angle of attack. Another major difference in the results of the two grids is the decrease in the critical Mach number for which the transition between separated and attached flow states occurs from $M=0.93$ for the baseline grid to $M=0.92$ for the fine grid. To put these load increments resulting from the bi-modal state into perspective, they are compared to the primary forces and moments for a non-zero angle of attack case, namely, the baseline grid at $M=0.9$ and $\alpha=2.5^{\circ}$, Table 3. With respect to this reference case normal force, the side force is seen to decrease by as much as $12 \%$ with the normal force increasing by the same amount. Comparing yaw and pitching moments to the reference pitching moment indicates a relative increase in pitching moment of up to $10 \%$ and yawing moment of $6 \%$. With the exception of axial force and rolling moment, the baseline grid produced the greatest bi-modal induced load increments.

In summary, the effect of the bi-modal transition from separated to attached flow creates an abrupt change of significant magnitude, Table 3 , in integrated forces and moments. However, there remains significant uncertainty in the values of the loading increment due to grid resolution.

\section{B. Line Loads}

The axial distributions of the integrated forces and moments were examined for all cases, however, for brevity only the line loads for Mach numbers 0.925 and 0.935 are included in Figures 8-13. The integration of the line loads with respect to $X$ recovers the fully integrated coefficients presented in the previous section. As before, results for the baseline grid are plotted in solid red, while results for the fine grid are in dashed black. An outline of the vehicle is also shown for reference. The bi-modal region, $777 \mathrm{in.} \leq X \leq 1100 \mathrm{in}$., which includes the full extent of the separated region, is highlighted in yellow. The axial point distributions chosen to form the $\Delta X$ integration bands are based on node locations of the finite element structural model.

\section{Axial Line Loads, $\Delta C_{X} / \Delta X$}

The axial force distributions for the baseline and fine grids, Figure 8, are nearly identical with the exception of the bi-modal region. This similarity reflects the relatively small differences of three to five percent seen in $C_{X}$ in Table 2 before and after the bi-modal flow state respectively. Another prominent feature of sectional $C_{X}$ plots is that negative 
Table 2. Integrated force and moment coefficient percent changes ${ }^{\mathrm{a}}$ between $^{-}$ fine and baseline grids.

\begin{tabular}{crrrrrr}
\hline \hline$M$ & $C_{X}(\%)$ & $C_{Y}(\%)$ & $C_{Z}(\%)$ & $C_{M X}(\%)$ & $C_{M Y}(\%)$ & $C_{M Z}(\%)$ \\
\hline 0.900 & 3.4 & -11.1 & 46.5 & 31.7 & -9.2 & -127.9 \\
0.920 & 3.8 & -17.8 & 35.4 & 29.5 & -3.9 & -130.1 \\
0.925 & 20.6 & -88.4 & -377.9 & -124.4 & 35.5 & 175.5 \\
0.930 & 20.5 & -84.4 & -493.5 & -106.6 & 40.3 & 374.5 \\
0.935 & 8.4 & -114.4 & -38.1 & -73.5 & -19.2 & -8.9 \\
0.940 & 9.3 & -150.4 & -78.4 & -80.3 & -75.1 & -94.8 \\
0.945 & 5.3 & -52.4 & 74.1 & -54.7 & -60.9 & -90.7 \\
0.950 & 5.0 & -57.2 & $-1623.7^{\mathrm{b}}$ & -52.6 & -53.0 & -88.1 \\
\hline \hline
\end{tabular}

a Percent change defined here as (fine - baseline) / baseline $\times 100 \%$

$\mathrm{b}$ The large percent change is due to the reference value being nearly zero.

Table 3. Bi-modal induced load coefficient increments, $\Delta C$, compared to non-zero angle of attack $^{\mathrm{a}}$ primary forces and moments for baseline ${ }^{\mathrm{b}}$ and fine grid $^{\mathrm{c}}$ cases.

\begin{tabular}{lrrrrrr}
\hline \hline Case & $\Delta C_{X}$ & \multicolumn{1}{c}{$\Delta C_{Y}$} & $\Delta C_{Z}$ & $\Delta C_{M X}$ & $\Delta C_{M Y}$ & $\Delta C_{M Z}$ \\
\hline Baseline Grid Case Increment (\%) $^{\mathrm{d}}$ & 11.8 & $-11.8^{\mathrm{f}}$ & 11.8 & -34.1 & 10.2 & $6.3^{\mathrm{g}}$ \\
Fine Grid Case Increment (\%) $^{\mathrm{d}}$ & 16.5 & $-4.9^{\mathrm{f}}$ & 7.0 & -163.1 & 4.0 & $5.3^{\mathrm{g}}$ \\
\hline Percent Change (\%) $^{\mathrm{e}}$ & 40 & -59 & -41 & 378 & -61 & -15 \\
\hline \hline
\end{tabular}

${ }^{\mathrm{a}} M=0.900, \alpha=2.5^{\circ}$, baseline grid.

${ }^{\mathrm{b}}$ Forces at $M=0.935$ minus forces at $M=0.930$. Moments at $M=0.940$ minus moments at $M=0.930$

${ }^{c}$ All values at $M=0.925$ minus values at $M=0.920$

${ }^{\mathrm{d}} \Delta C / C_{\operatorname{Ref}} \times 100 \%$ except for $\Delta C_{Y}$ and $\Delta C_{M Z}$, which are compared to reference normal force and pitching moment respectively.

${ }^{\text {e }}$ Percent change defined here as (fine - baseline) $/$ baseline $\times 100 \%$

f $\Delta C_{Y} / C_{Z \operatorname{Ref}} \times 100 \%$

${ }^{\mathrm{g}} \Delta C_{M Z} / C_{M Y \operatorname{Ref}} \times 100 \%$

regions are indicative of flow separation, hence the large troughs seen in the bi-modal region over the SM and SA. Once the flow attaches the remaining small dip reflects the local separation due to the RCS.

\section{Side Line Loads, $\Delta C_{Y} / \Delta X$}

Due to symmetry, the side line loads, Figure 9, approach zero except in the areas of protuberances. Relatively large changes are noted due to the asymmetric separation present in the bi-modal state. At $M=0.925$ it is evident from Figure 9 that the fine grid is supporting a weakly bi-modal state, while the baseline grid solution doesn't begin its stronger asymmetric reattachment until $M=0.935$.

\section{Normal Line Loads, $\Delta C_{Z} / \Delta X$}

At zero incidence, normal line loads, Figure 10, also approach zero except in regions of asymmetry. Compared to the local side force, the local normal force increment due to the bi-modal flow is larger for the fine grid, Figures 9 and 10, while smaller for the baseline grid. This difference in force increment indicates that the fine grid bi-modal state is oriented more towards the normal direction, $Z$, while the baseline grid bi-modal area is stronger in side force, $C_{Y}$. This effect is somewhat masked when comparing the load increments for the fully integrated loads in Table 3 due to the canceling of positive and negative force contributions. 


\section{Rolling Moment Line Loads, $\Delta C_{M X} / \Delta X$}

Due to symmetry, rolling moment line loads, Figure 11, tend to zero even for non-zero angles of attack. Like axial line loads, the sign change is indicative of reattachment. However, unlike the axial line load results, differences between grids can easily be seen in the frustum and aft skirt.

\section{Pitching Moment Line Loads, $\Delta C_{M Y} / \Delta X$}

Due to symmetry, rolling moment line loads, Figure 11, tend to zero even for non-zero angles of attack. Like axial line loads, the sign change is indicative of reattachment. However, unlike the axial line load results, differences between grids can easily be seen in the frustum and aft skirt. Due to the moment reference center being located at the nozzle gimbal point, Figure 1, the pitching moment correlates strongly with normal force, see Figure 12. Local peak values of sectional pitching moment are approximately the same for each grid, with the fine grid peaking earlier by a Mach number offset of 0.01 .

\section{Yawing Moment Line Loads, $\Delta C_{M Z} / \Delta X$}

Yawing moment correlates strongly with side force, see Figure 13. As with the side force plots of Figure 9, the yawing moment is seen to be much larger for the baseline grid.

\section{Surface Pressures}

Continuing to widen the scope of view, surface pressures are plotted for Mach numbers (from 0.920 to 0.935), Figure 14. In order to visualize the whole region of interest in one plot, the surface is unrolled by transforming the grid into arc length $(r \phi)$ verses axial station $(X)$ coordinates. Specific locations on the surface are referenced by their axial station and lines of constant $\phi$. From these plots the mechanism of the bi-modal phenomenon can be seen to be the result of a remaining pocket of separated flow surrounding the CM-SM umbilical near $X \approx 800 \mathrm{in}$. and $\phi \approx 22^{\circ}$. At $M=0.925$ the baseline grid solution remains fully separated, however, for the fine grid the flow has nearly completely reattached, as seen by purple contours of lowered pressure, with only a small region of separation near the umbilical remaining. Another striking feature of the reattachment is the abruptness of the change going from $M=0.920$ to $M=0.925$ for the fine grid and $M=0.930$ to $M=0.935$, for the baseline grid.

\section{Flow Field}

The relationship of the off surface flow field to the surface pressures and simulated surface oil flow is plotted in Mach number contours, Figure 15, and simulated schlieren, Figure 16, at several sample free-stream Mach numbers. By comparing the cuts at $\phi=0^{\circ}$ and $\phi=45^{\circ}$ for $M=0.935$, Figures 3(a) and 15(c), the effect of the wake of the LAS nozzles on the flow over the SM can be seen. Namely, that the lower speed wake flow results in a lowered acceleration of the attached flow around the CM-SM junction and a weaker terminating shock. The pressure signature of this decrease in flow acceleration can be seen in the corresponding unrolled surface pressure plot at $\phi=-90^{\circ}$ and $180^{\circ}$, Figure 14(g).

Comparing cutting planes at $\phi=0^{\circ}$ for both grids shows that other than differences of when the bi-modal event occurs, the flow fields are very similarly resolved. At $M=0.920$, the Mach number flow field contours are very nearly the same with the main difference being the stronger wake from the LAS nozzles for the fine grid. The simulated schlieren at this same condition, Figure 16, also reveals a more sharply resolved LAS wake structure. After reattachment at $M=0.95$, Mach number contours of both grids are again very similar. In the simulated schlieren view at the same Mach number, the effect of the out of family grid refinement can be seen, with the baseline grid solution having a sharper shock structure near the surface of the SM and the fine grid solution better resolved further away. This should not be confused with the better resolution of the fine grid along the CM-SM junction ridge, which is more critical for accurate prediction of flow separation or attachment, recall Figure 6.

\section{Dynamic Aeroelastic Results}

The abrupt transition from separated to attached flow around the CM-SM junction with increasing Mach number demonstrates the instability of the flow with respect to Mach number. The point of instability is also dependent on cone angle and angle of attack. ${ }^{1,2}$ Defining the critical Mach number, $M_{c}$, from which the flow transitions from fully 
separated to attached and examining Figure 3 in Erickson ${ }^{3}$ the following trends are identified: 1) at a constant angle of attack, $M_{c}$ increases with increasing cone angle, 2) at a constant cone angle, increasing angle of attack increases $M_{c}$ on the leeward side while decreasing $M_{c}$ on the windward side, due to the respective thickening and thinning of boundary layers. In other words, at a particular Mach number, increasing the cone angle makes the flow more likely to separate, while increasing angle of attack makes the lee side more likely and the windward side less likely to separate. It should be noted that in wind tunnel tests ${ }^{1,2,24}$ there is a range of Mach number and angle of attack that will exhibit the alternating flow instability. This instability can be plotted as islands of alternating flow with respect to $M$ and $\alpha$ for a constant cone half angle, $\theta_{c}{ }^{3}$ Thus, it is this sensitivity to angle of attack that prompts the question of whether the local change in $\alpha$ resulting from vehicle flexibility would be enough to trigger and sustain the alternating flow phenomenon.

The dynamic aeroelastic analysis was performed on the fine grid at two Mach numbers which bracket the onset of the flow transition from separated $(M=0.920)$ to attached $(M=0.925)$. From the generalized displacement history of the modes included in the analysis at $M=0.920$, the first six of which are shown in Figure 17, it is seen that with the given generalized modal velocity perturbation of 500, all modal displacements are damped. Also plotted in black is the response of the structure without the influence of aerodynamics. Because of the large initial velocity, the response is dominated by elastic restoring forces rather than interactions with the aerodynamics. Normal and lateral displacement histories of the LAS tip, plotted in Figure 18, indicate the upper bound of the displacement of the vehicle due to the initial perturbation is about 17 inches. Note that the choice of the initial generalized velocity is arbitrary and is intentionally chosen to be very large to serve as an upper bound for determining sensitivity of the bi-modal state to vehicle motion. Inspection of an animated history of surface pressures, a single frame of which is shown in Figure 19, indicates that at $M=0.92$ there is no change in the flow state from separated to attached. This is not surprising given that the maximum displacement of approximately five inches in the CM-SM region results in only a $0.14^{\circ}$ change in local angle of attack. Note, the much larger tip displacements of the LAS are due to the forced excitation of the higher mode shapes. The dynamic analysis was repeated for the attached flow state at $M=0.925$. Generalized displacement histories, Figure 20, again showed all modes were damped. Tip displacement histories, Figure 21 show the same magnitude in tip displacement, however, due to differences in the excitation, the peak is seen to be in the normal component rather than the lateral. The difference in excitation reflects the sign of the mode shapes which is arbitrary and does not affect the natural vibration response due to same physical excitation. Again by examining the animated history of surface pressures, a single frame of which is shown in Figure 22, the flow state was seen to remain fully attached throughout the perturbation. Thus, it is concluded that the bi-modal flow field is insensitive to deflections larger than any expected vehicle motions due to aeroelastic effects.

\section{Effect of Angle of Attack}

Computations were run at incidence on the baseline grid without aeroelastic effects. As cited in the previous section, the transition from separated to attached flow around the CM-SM junction is known to be a function of angle of attack. Though the aeroelastic deflections resulted in exceedingly small induced local angles of attack, the nominal trajectory may include angles as large as $2.5^{\circ}$, therefore it is important to quantify the bi-modal loading under this condition.

As in the zero incidence case, increments in the total integrated forces and moments are normalized by a reference condition of $M=0.900$ and $\alpha=2.5^{\circ}$, in Table 4. With respect to this reference case normal force, the side force is seen to decrease by only $1.6 \%$, compared to the $11.8 \%$ of the zero incidence case with the accompanying yawing moment delta also an order of magnitude smaller than the zero incidence case. While the normal force delta is slightly lower than the zero incidence case, the pitching moment delta of $27.4 \%$ is nearly triple that of the zero incidence case of $10.2 \%$ for the same grid, see Table 3. Thus, the effect of angle of attack on the bi-modal phenomenon is to reduce the lateral increment while significantly increasing the longitudinal increment.

Another effect of angle of attack is the reduction in the Mach number value at which the bi-modal state starts by 0.015, as defined by the beginning of the sharp rise in pitching moment on the baseline grid in Figures 7(d) and 23(b). The magnitude of the normal force increment resulting from the bi-modal state decreases slightly to $9.4 \%$, while the pitching moment increment nearly triples to $27 \%$. Thus, the bi-modal effect on pitching moment is seen to be significantly amplified by a change in the angle of attack that might be encountered in the flight trajectory.

Though the numerical labels have been redacted in the plots in Figure 23, the left and right scales are the same in each plot, with the origin offset to better capture the relative changes in the quantities. A feature of particular interest in these plots is the sinusoidal-like fluctuation of normal force and pitching moment through the bi-modal event. This sinusoidal fluctuation can be understood by examining the surface pressure plots in Figure 24, which show the unrolled 
surface pressure plots (left) and the side view of the flow field Mach number and surface pressure contours (right) at key free-stream Mach numbers. Namely, $M=0.880$ where the flow is fully separated, $M=0.915$ and $M=0.935$ where minimum and maximum values of $C_{M Y}$ occur respectively, and $M=0.950$ where the flow is fully attached around the CM-SM junction. The sinusoidal-like trend with increasing Mach number is due to a more gradual transition from separated to attached flow in the bi-modal state where the decrease in pressure due to attachment is weighed against replacement of the relatively low pressure separation bubble downstream with the high pressure resulting from the shock caused by the expansion corner. This pressure distribution causes a shift from initially being a net loss of pitching moment to a net gain. For example, at $M=0.915$, Figure 24(c), in the lower quadrant centered around $\left(\phi=180^{\circ}\right)$, the shock is weak, so the pressure rise is minimal. Compare that with $M=0.935$, Figure 24(e), where the pitching moment peaks, and one can see the higher pressures after the shock everywhere except for the remaining separation bubble centered at approximately $\phi=30^{\circ}$. Once this low pressure area is gone, Figure 24(g), the pitching moment temporarily drops before resuming its upward trend with Mach number, Figure 23.

Table 4. Bi-modal induced load coefficient increments, $\Delta C$, between $M=0.915$ and $M=0.935$ at $2.5^{\circ}$ angle of attack as a percentage their respective values at $M=0.900^{\text {a }}$ except where noted.

\begin{tabular}{|c|c|c|c|c|c|}
\hline$\Delta C_{X}(\%)$ & $\Delta C_{Y}{ }^{\mathrm{b}}(\%)$ & $\Delta C_{Z} \quad(\%)$ & $\Delta C_{M X}$ & $\Delta C_{M Y} \quad(\%)$ & $\Delta C_{M Z^{\mathrm{c}}}(\%)$ \\
\hline 8.3 & -1.6 & 9.4 & 29.9 & 27.4 & -0.6 \\
\hline
\end{tabular}

\section{Concluding Remarks}

A Reynolds averaged Navier-Stokes analysis, including dynamic aeroelastic effects, was performed on the Ares I-X launch vehicle at transonic Mach numbers and flight Reynolds numbers for two grid resolutions and two angles of attack to examine the nature of its bi-modal flow field. The bi-modal flow phenomenon is of interest to the guidance, navigation and control community because it causes a discontinuous jump in forces and moments. Zero angle of attack results of the rigid structure on the baseline grid, expressed as a percentage of the normal force and pitching moment at $M=0.9, \alpha=2.5^{\circ}$, indicate a jump in normal and side forces of $12 \%$ with a pitching moment increment of $10 \%$.

Fine grid dynamic aeroelastic analysis at zero incidence did not show any aeroelastic instability and did not show any change in flow separation due to the very small changes seen in the local angle of attack of $0.14^{\circ}$. Thus, it is concluded that the bi-modal flow field is insensitive to deflections larger than any expected vehicle motions due to aeroelastic effects.

At angle of attack, the bi-modal phenomenon is seen to significantly increase the pitching moment increment to nearly triple that of the zero incidence case for the same baseline grid. While rises are seen in axial force and rolling moment for all cases, these are of less interest because an increment in drag does not present a control issue and the absolute values of rolling moment for these cases are exceedingly small and grid sensitive.

Significant grid-induced variations in the flow field solutions indicate that further grid refinement is warranted, though the finer grid was seen to have a much smaller bi-modal surface area and a normal force increment of about half due to a more uniform transition from fully separated to fully attached flow over the service module. The most likely reason for this variation in the transition to separation is the increased refinement in the region of the conecylinder junction where the flow separation begins.

\section{Acknowledgments}

The authors gratefully acknowledge Russ Rausch for proposing this study and the full Ares aeroelastic analysis team for their guidance and discussions. Grids used in this study were created by Raymond Mineck of ATK, Space Division. The authors would also like to acknowledge the Ares I-X and Ares I project offices for their support. 


\section{References}

${ }^{1}$ Chevalier, H. L. and Robertson, J. E., "Pressure Fluctuations Resulting from an Alternating Flow Separation and Attachment at Transonic Speeds," Tech. Rep. AEDC-TDR-63-204, Nov. 1963.

${ }^{2}$ Robertson, J. E., "Unsteady Pressure Phenomena for Basic Missile Shapes at Transonic Speeds," AIAA Paper 1964-0003, Jan. 1964.

${ }^{3}$ Ericsson, L. E., "Aeroelastic Instability Caused by Slender Payloads," J. Spacecraft, Vol. 4, No. 1, 1967, pp. pp 65-73, Also, AIAA Paper 1966-0471.

${ }^{4}$ Ericsson, L. E., "Effects of shock induced boundary layer separation on cone cylinder body aerodynamics," Tech. Rep. L-87-67-2, Lockheed Missiles \& Space Company, 1967, Also, NASA-CR-61560.

${ }^{5}$ Baker, R. L., "A Historical Perspective on the Buffet/Acoustic Environments of the Titan IV Launch Vehicle," AEROSPACE REPORT TOR-2005(1413)-1, El Segundo, CA 90245-4691, Sept. 2005.

${ }^{6}$ Streett, C. L., "Surface Pressure Fluctuation Estimates Guided by CFD," Jan. 2008, Unpublished Ares project presentation.

${ }^{7}$ Sekula, M., Piatak, D., and Rausch, R., "Analysis of a Transonic Alternating Flow Phenomenon Observed During Ares Wind Tunnel Tests," AIAA Paper 2010-4370, 2010.

${ }^{8}$ Piatak, D., Sekula, M., and Rausch, R., "Ares Launch Vehicle Transonic Buffet Testing and Analysis Techniques," AIAA Paper 2010-4369, 2010.

${ }^{9}$ Massey, S. J. and Chwalowski, P., “CFD Analysis of Ares I-X Bi-Modal Loading,” NASA Ares-AE-TA-0008, March 2009.

${ }^{10}$ Pirzadeh, S. Z., "Advanced Unstructured Grid Generation for Complex Aerodynamic Applications,” AIAA Paper 2008-7178, Aug. 2008.

${ }^{11}$ Samareh, J. A., "Unstructured Grids on NURBS Surfaces," AIAA Paper 1993-3454.

${ }^{12}$ Anderson, W. K. and Bonhaus, D. L., "An Implicit Upwind Algorithm for Computing Turbulent Flows on Unstructured Grids," Computers \& Fluids, Vol. 23, No. 1, 1994, pp. 1-21.

${ }^{13}$ NASA LaRC, Hampton, VA, FUN3D 10.6.0 Manual, Nov. 2008, http://fun3d.larc.nasa.gov.

${ }^{14}$ Spalart, P. R. and Allmaras, S. R., “A One-Equation Turbulence Model for Aerodynamic Flows,” La Recherche Aerospatiale, No. 1, 1994, pp 5-21.

${ }^{15}$ Edwards, J. R., "A Low-Diffusion Flux-Splitting Scheme for Navier-Stokes Calculations," AIAA Paper 1995-1703, 1995.

${ }^{16}$ Biedron, R. T. and Thomas, J. L., "Recent Enhancements To The FUN3D Flow Solver For Moving-Mesh Applications," AIAA Paper 2009-1360, Jan. 2009.

${ }^{17}$ Biedron, R. T. and Lee-Rausch, E. M., “Rotor Airloads Prediction Using Unstructured Meshes and Loose CFD/CSD Coupling," AIAA Paper 2008-7341, 2008.

${ }^{18}$ Batina, J. T., "Unsteady Transonic Flow Calculations for Realistic Aircraft Configurations,” AIAA Paper 1987-0850, 1987.

${ }^{19}$ Bartels, R. E., Rumsey, C. L., and Biedron, R. T., “CFL3D Version 6.4 - General Usage and Aeroelastic Analysis,” NASA TM 2006-214301, March 2006.

${ }^{20}$ MSC Software, Santa Ana, CA, MSC Nastran, 2008, http://www.mscsoftware.com/products/msc_nastran.cfm.

${ }^{21}$ Bartels, R., Chwalowski, P., Massey, S., Vatsa, V., Heeg, J., Mineck, R., and Wieseman, C., "Computational Aeroelastic Analysis of the Ares Crew Launch Vehicle During Ascent," AIAA Paper 2010-4374, 2010.

${ }^{22}$ Samareh, J. A., "Discrete Data Transfer Technique for Fluid-Structure Interaction,” AIAA Paper 2007-4309, June 2007.

${ }^{23}$ Tecplot, Inc., Bellevue, WA, Tecplot 3602008 User's Manual, 2008.

${ }^{24}$ Piatak, D. J., Florance, J. P., Ivanco, T. G., Rausch, R. D., Sekula, M. K., and Wieseman, C. D., "Test Summary Document for the 3.5 Percent Ares I-X Rigid Buffet Model,” NASA Ares-AE-TA-0002, June 2008. 


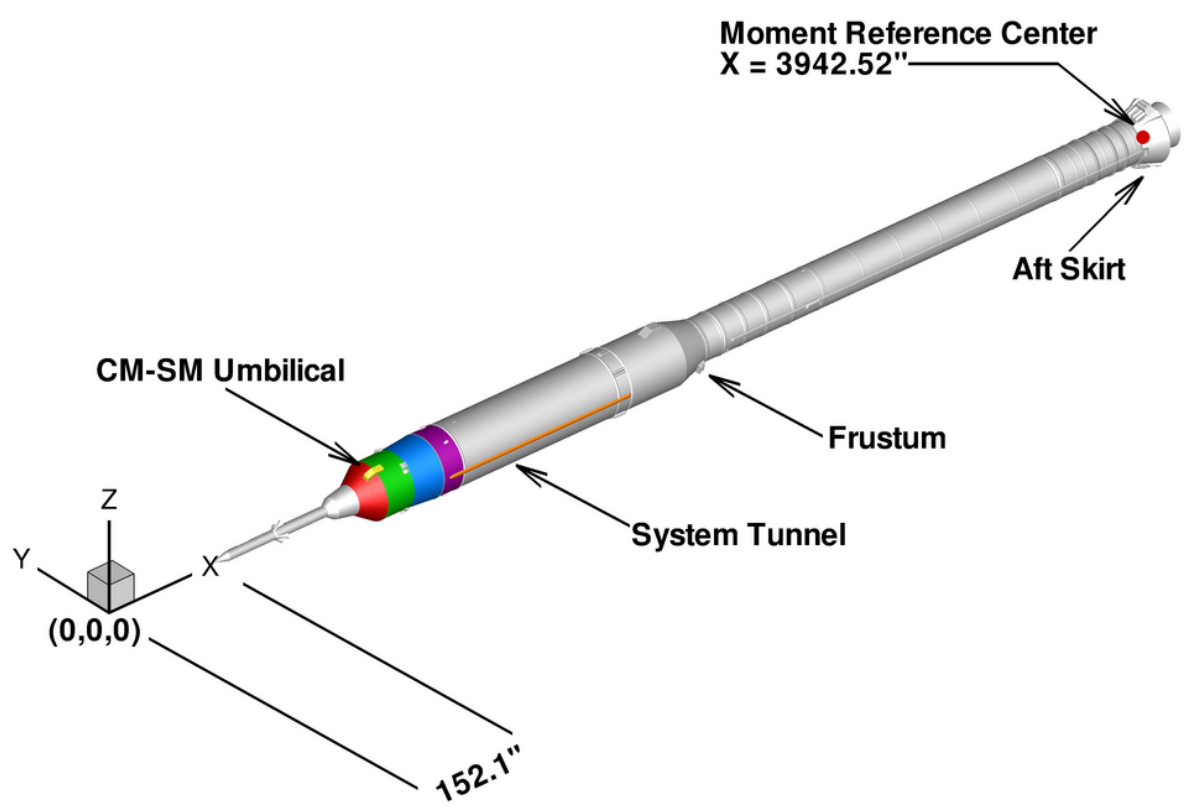

Figure 1. CFD axis orientation and moment reference center location for Ares I-X.

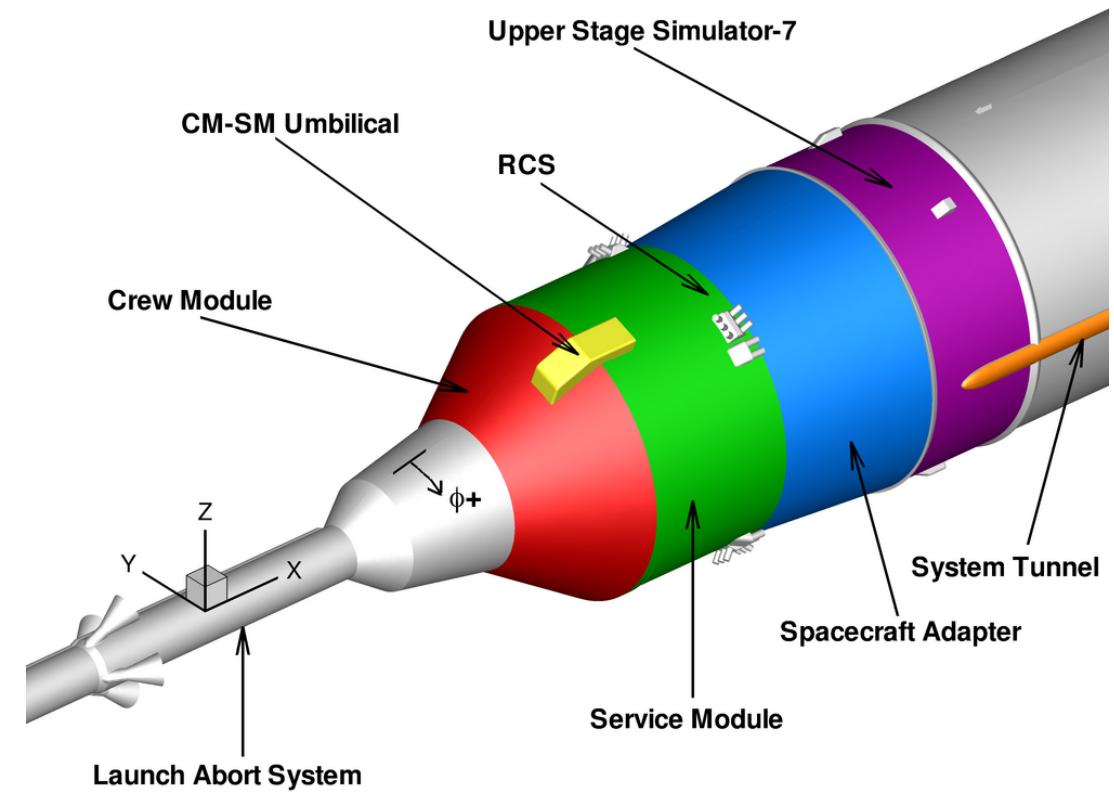

Figure 2. Ares I-X components in the bi-modal region and azimuthal coordinate, $\phi$ orientation. 


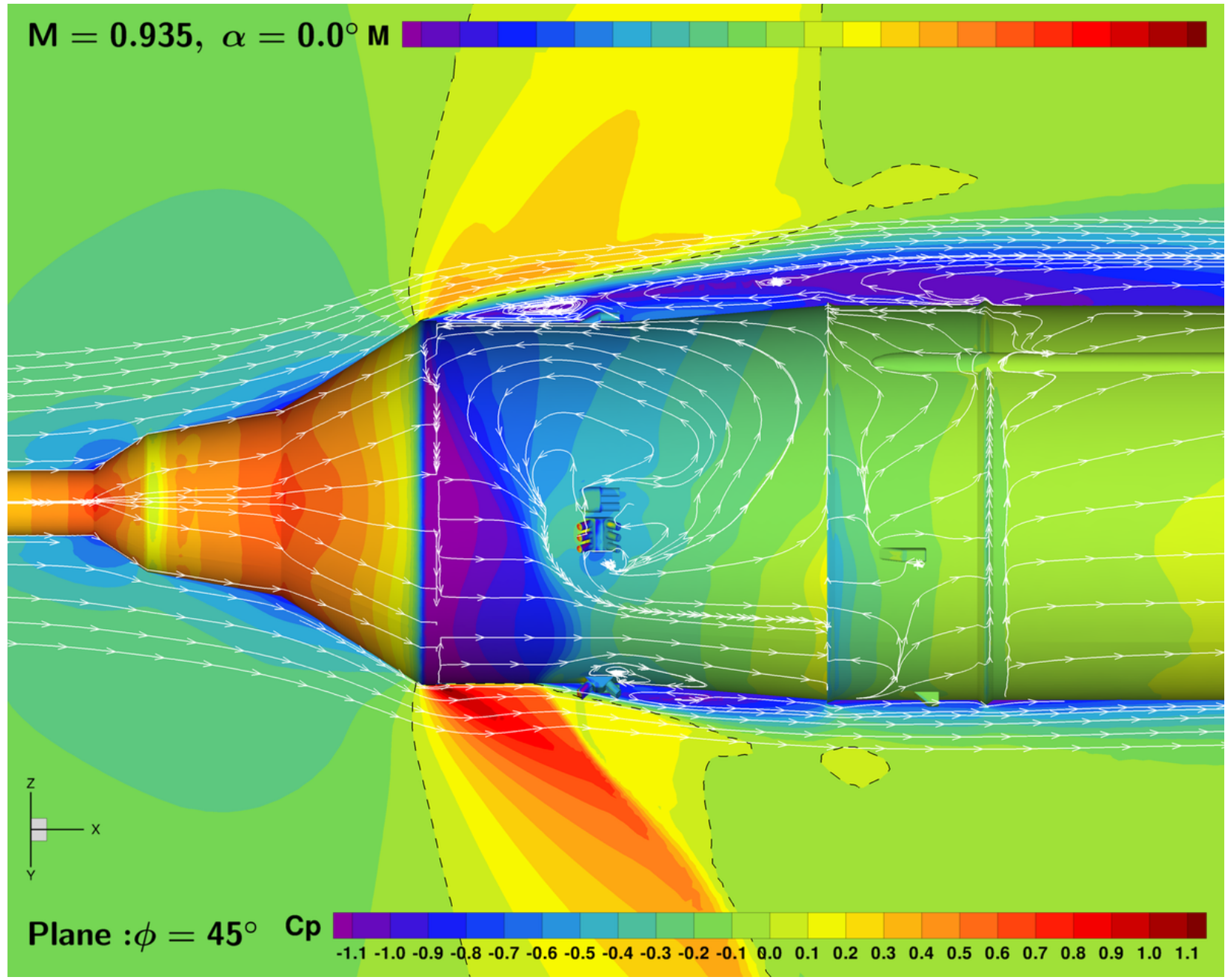

(a) Surface $C_{p}$ and Mach number on $\phi=45^{\circ}$ plane. Streamlines shown in white with arrows indicating flow direction.

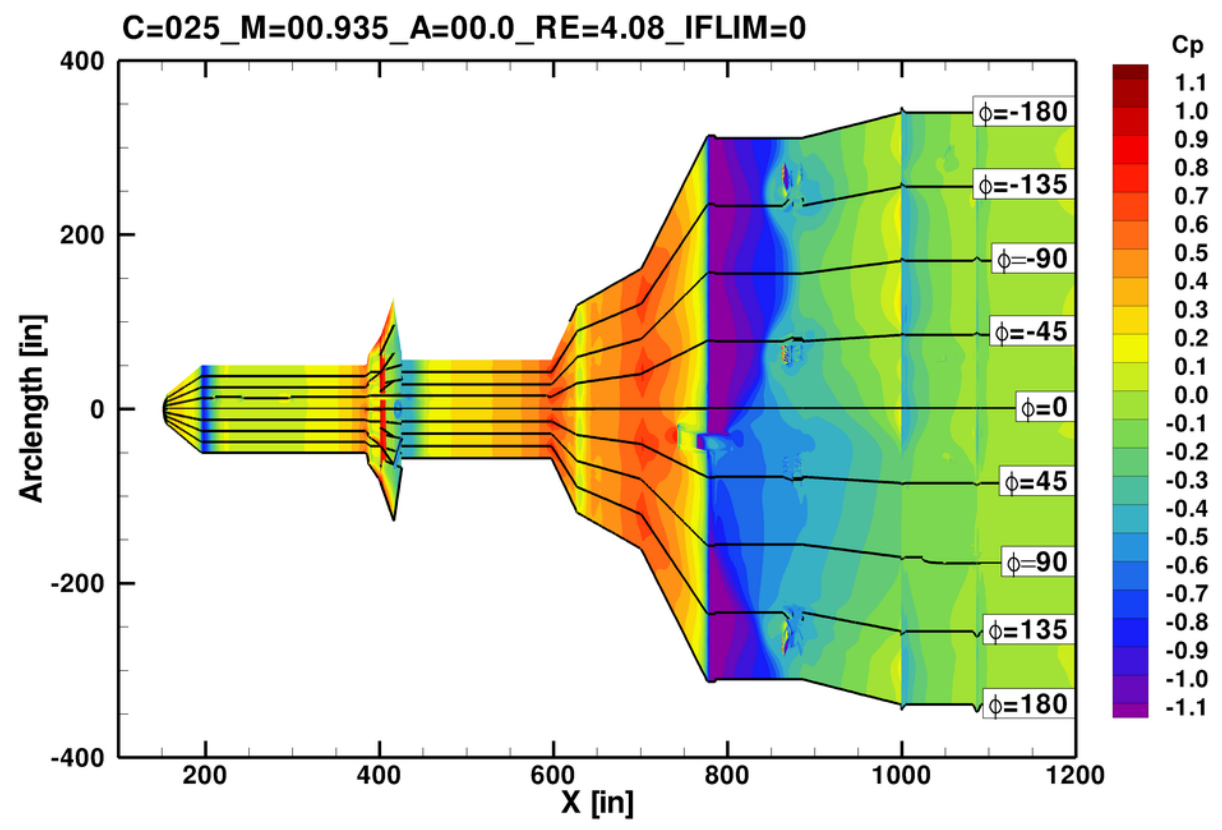

(b) $C_{p}$ on unrolled surface with constant $\phi$ lines in black.

Figure 3. Baseline grid, $M=0.935, \alpha=0^{\circ}$ : Example of bi-modal flow state. 


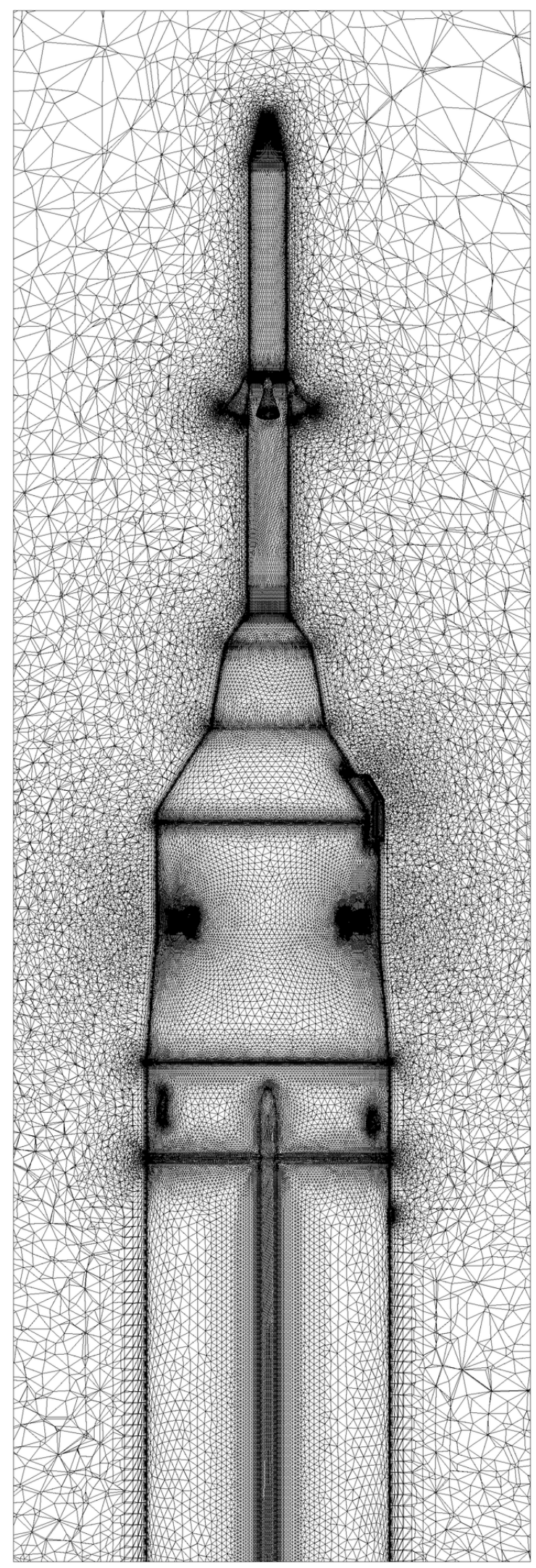

(a) 22 million nodes

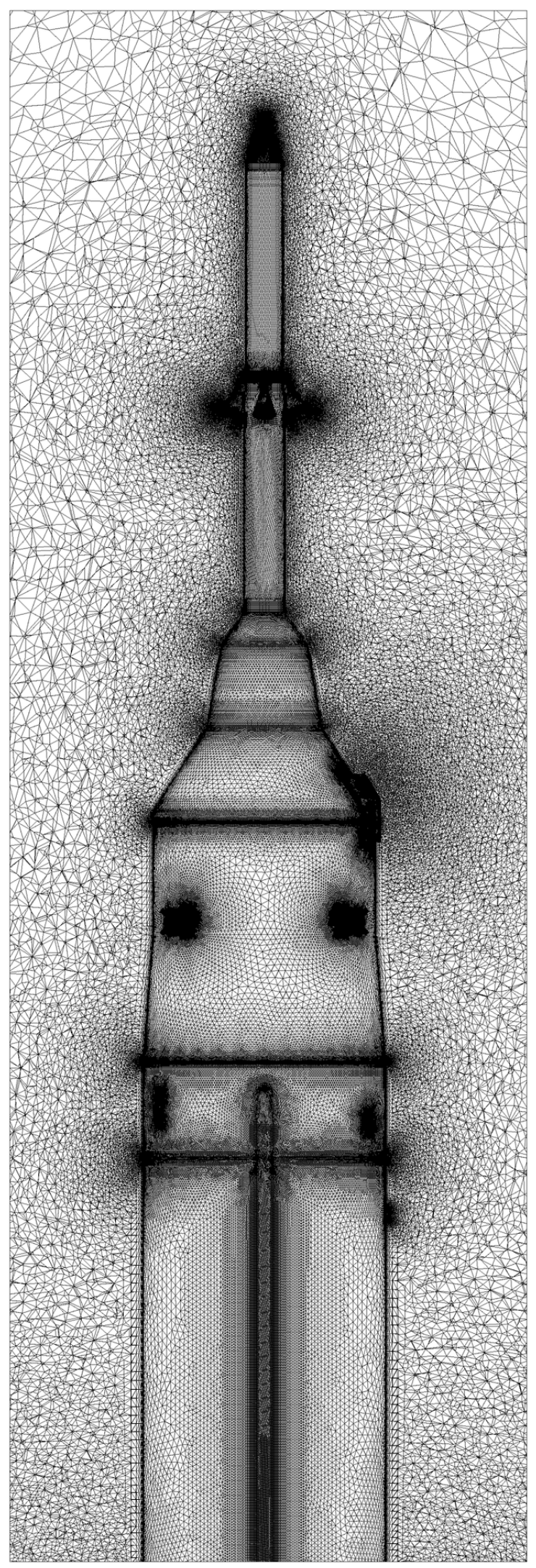

(b) 48 million nodes

Figure 4. Grid surface and $y=0$ plane for baseline and fine grids. 


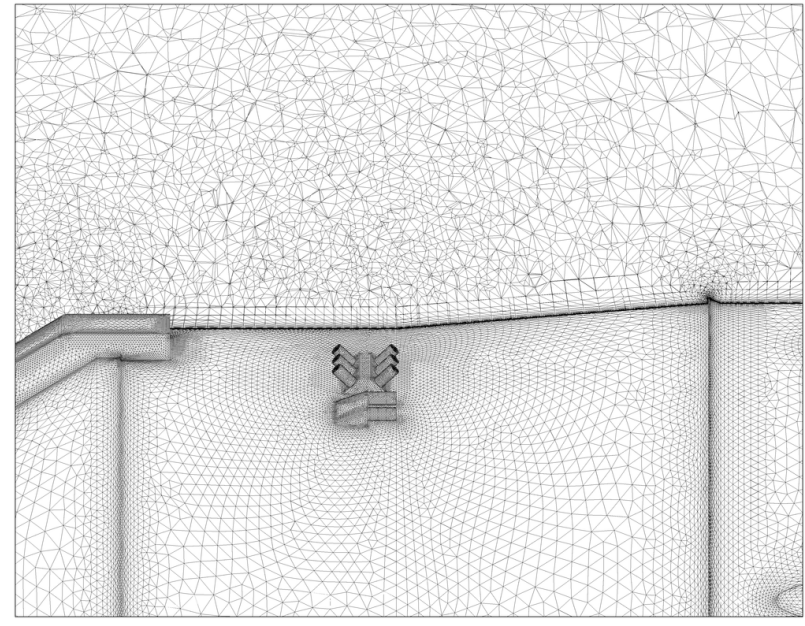

(a) 22 million nodes

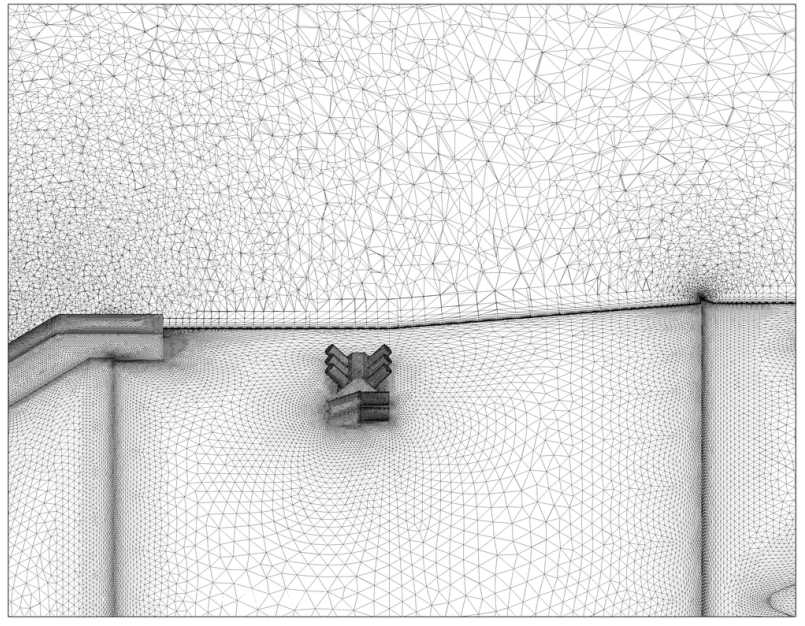

(b) 48 million nodes

Figure 5. Baseline and fine grids in flow separation region, $\phi=0^{\circ}$.

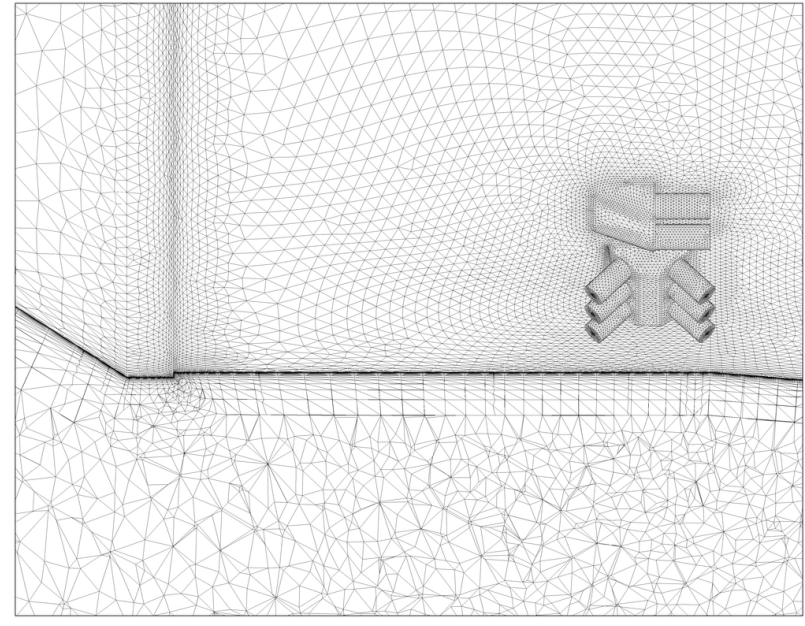

(a) 22 million nodes

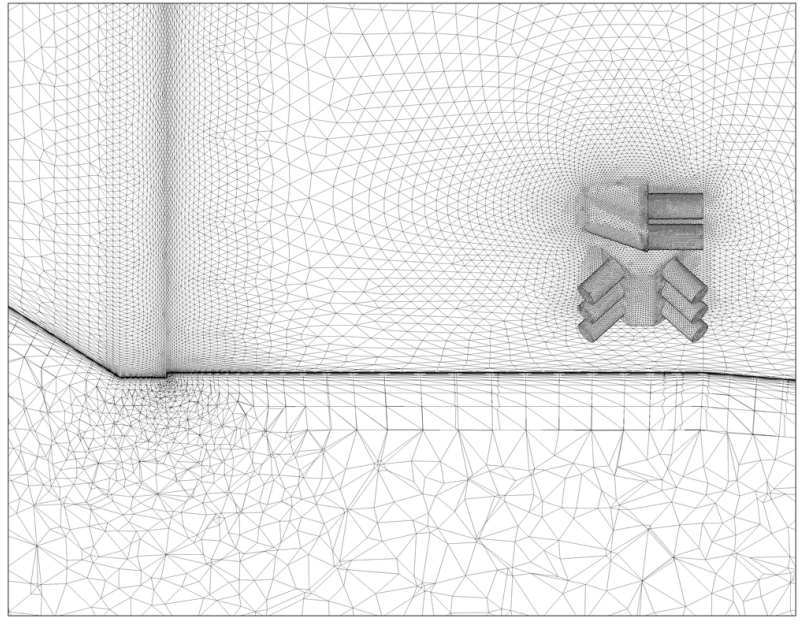

(b) 48 million nodes

Figure 6. Baseline and fine grids in cone-cylinder junction region, $\phi=180^{\circ}$. 


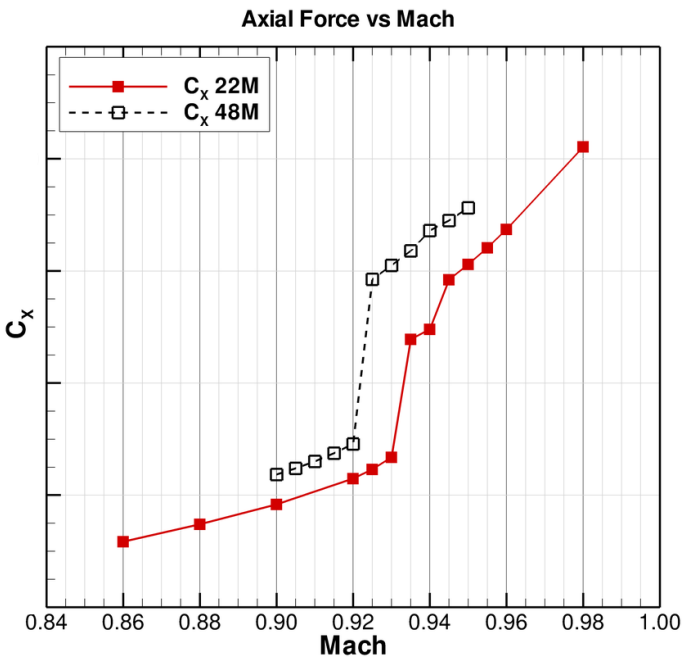

(a)

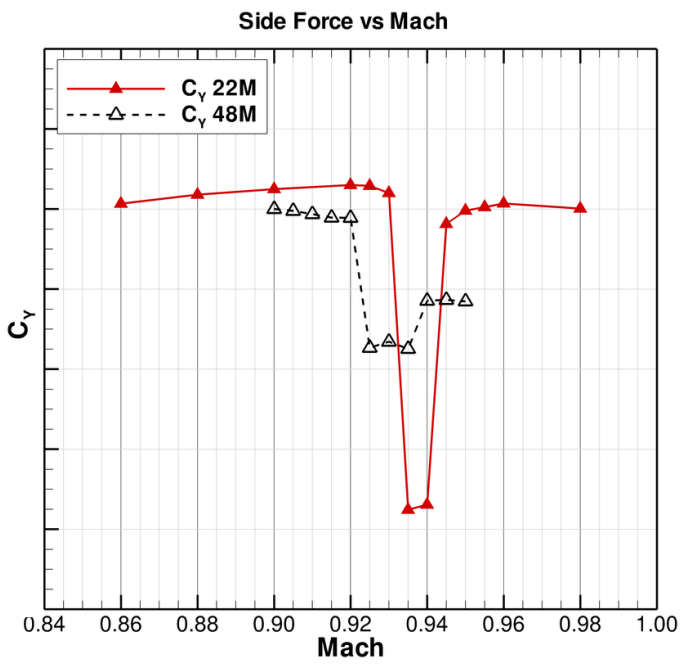

(c)

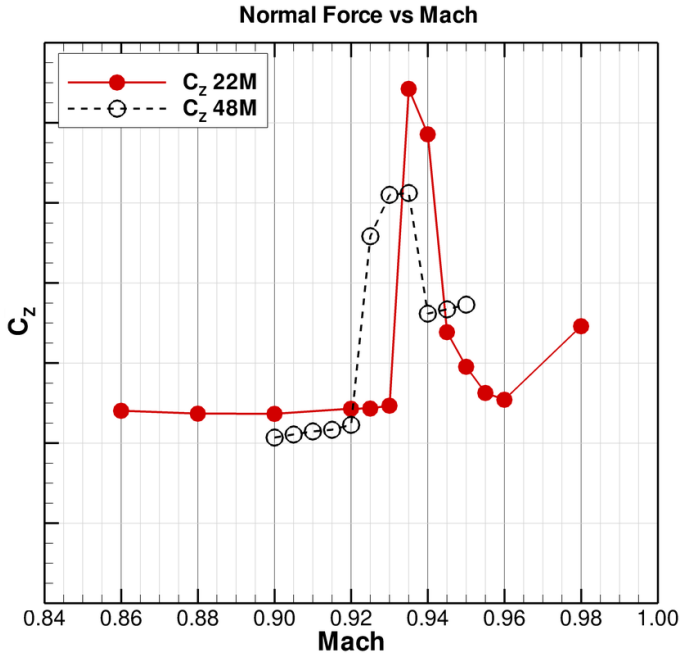

(e)

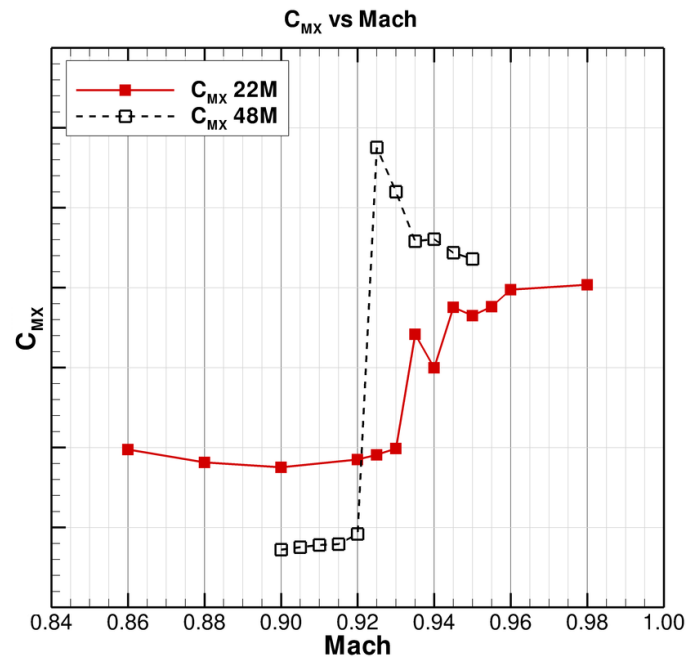

(b)

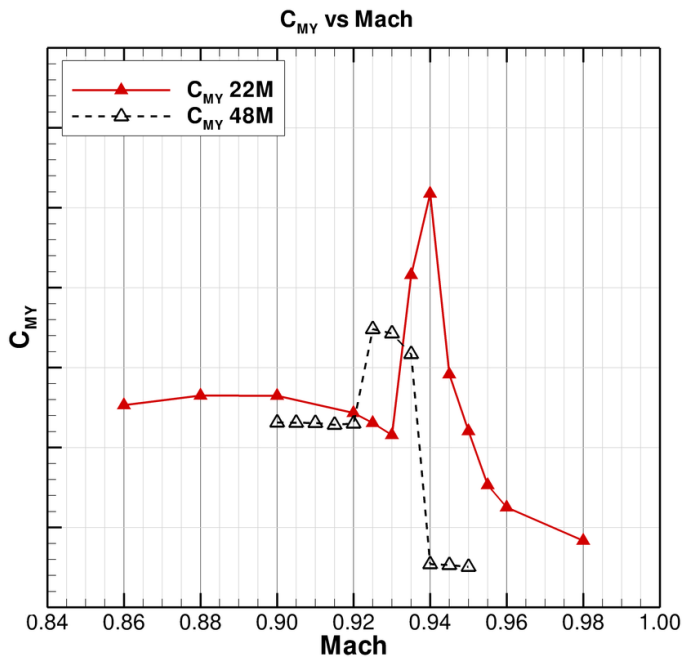

(d)

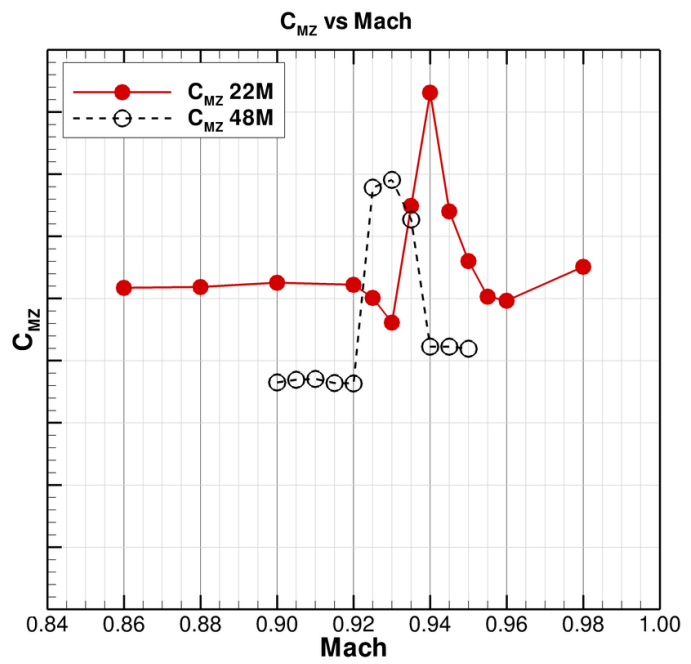

(f)

Figure 7. $\alpha=0^{\circ}$ : Integrated forces and moments for baseline (22M) and fine (48M) grids. 


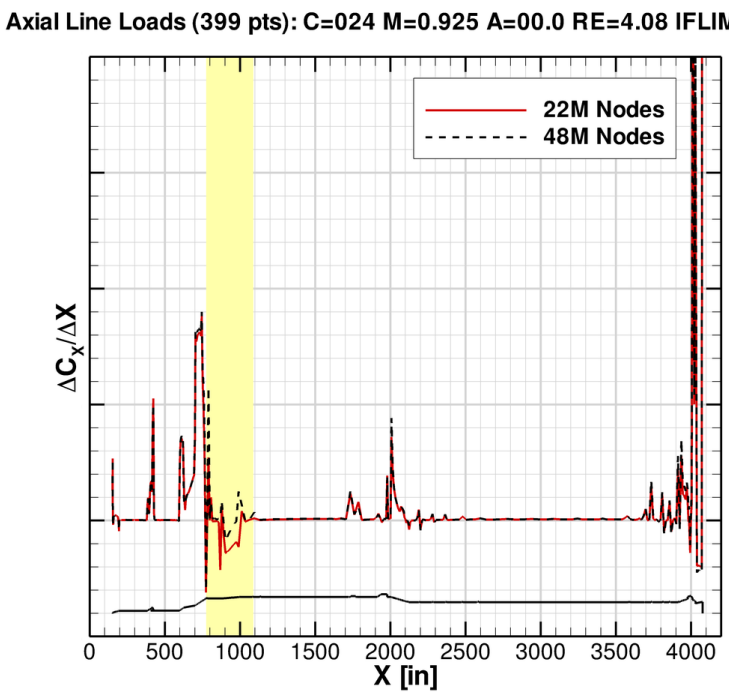

(a) $\mathrm{M}=0.925$
Axial Line Loads (399 pts): C=025 M=0.935 A=00.0 RE=4.08 IFLIM=0

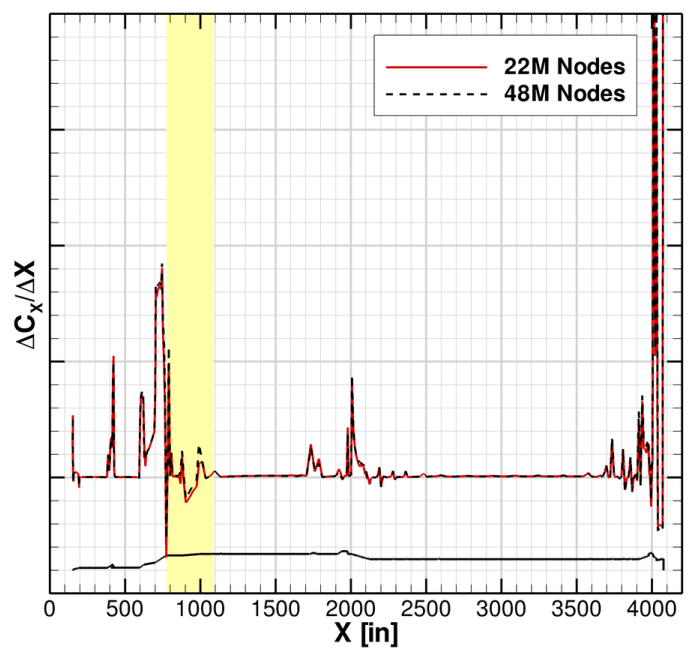

(b) $\mathrm{M}=0.935$

Figure 8. $\alpha=0^{\circ}$ : Axial force line loads for baseline and fine grids.

Side Line Loads: (399 pts) : C=024 M=0.925 A=00.0 RE=4.08 IFLIM=0

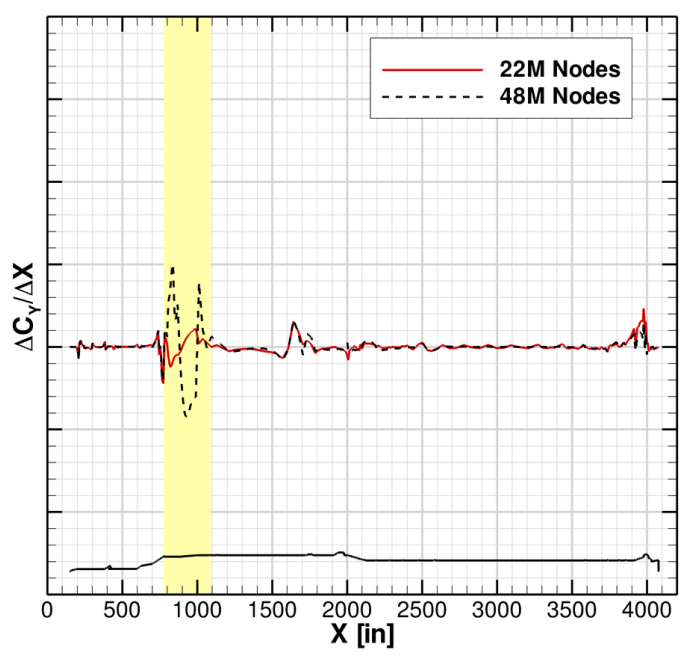

(a) $\mathrm{M}=0.925$
Side Line Loads: (399 pts): C=025 M=0.935 A=00.0 RE=4.08 IFLIM=0

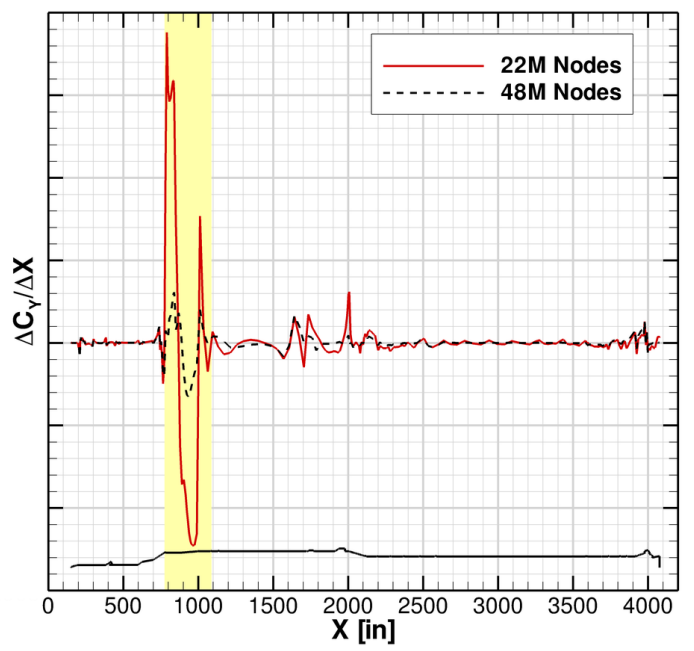

(b) $\mathrm{M}=0.935$

Figure 9. $\alpha=0^{\circ}$ : Side force line loads for baseline and fine grids. 


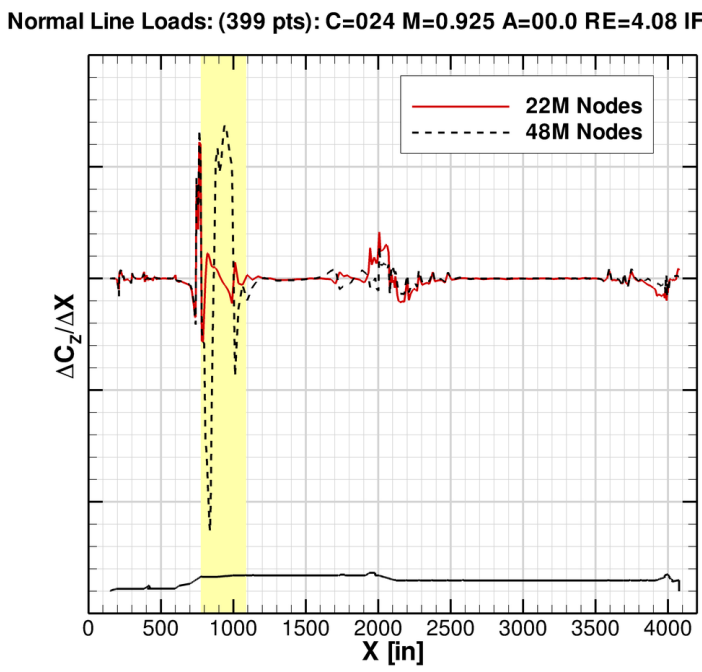

(a) $\mathrm{M}=0.925$

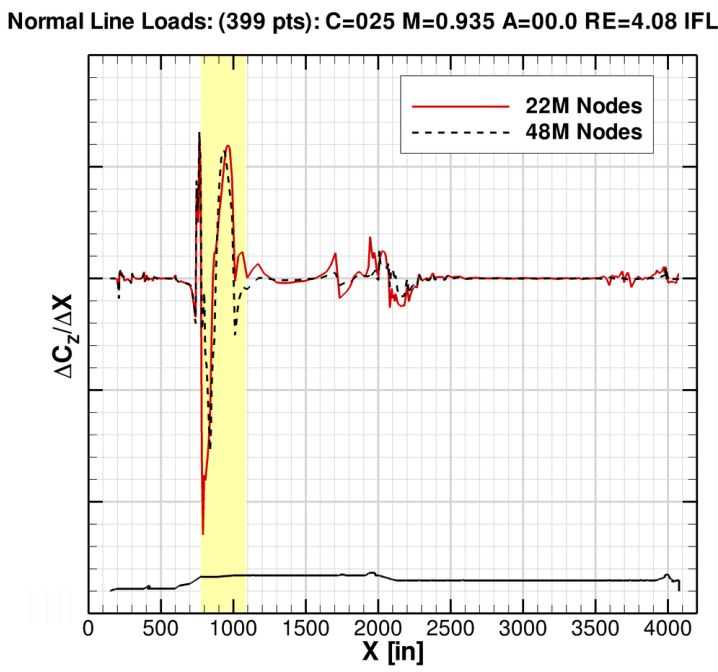

(b) $\mathrm{M}=0.935$

Figure 10. $\alpha=0^{\circ}$ : Normal force line loads for baseline and fine grids.

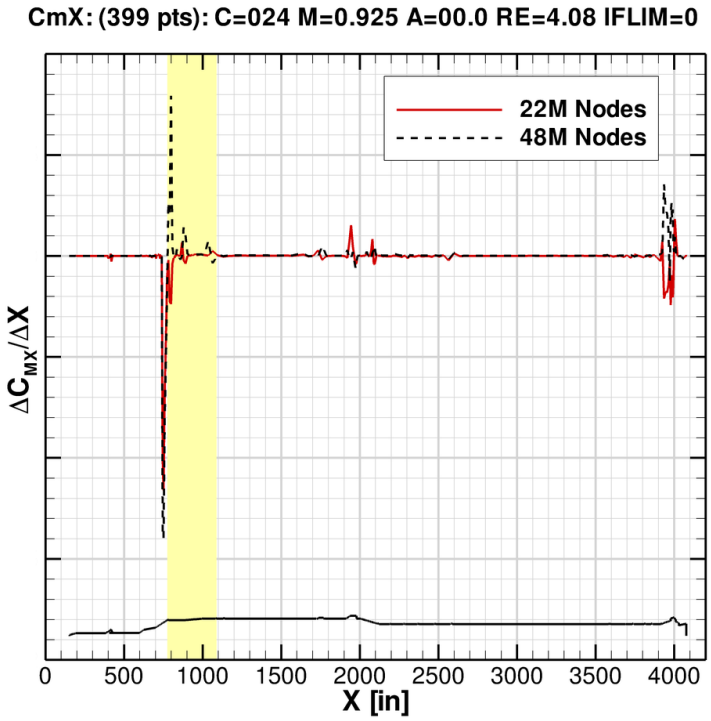

(a) $\mathrm{M}=0.925$

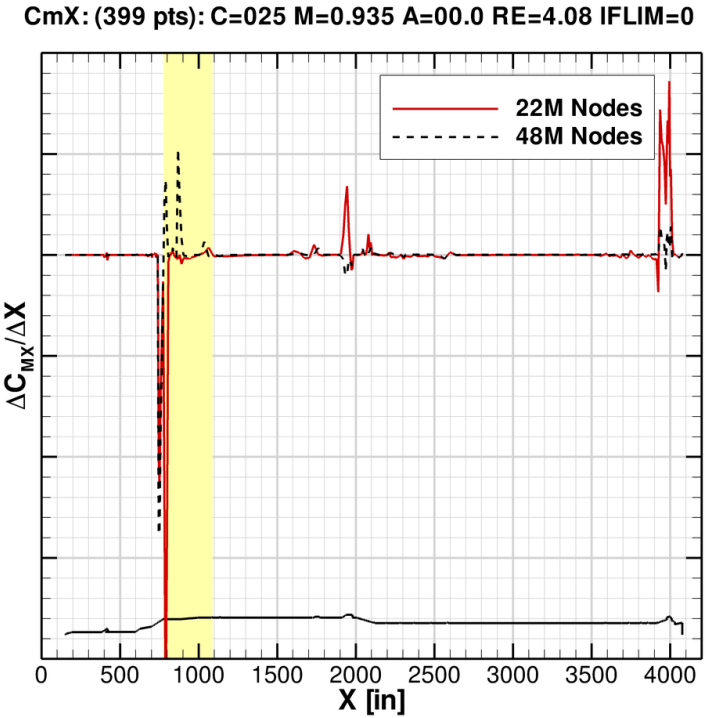

(b) $\mathrm{M}=0.935$

Figure 11. $\alpha=0^{\circ}$ : Rolling moment line loads for baseline and fine grids. 


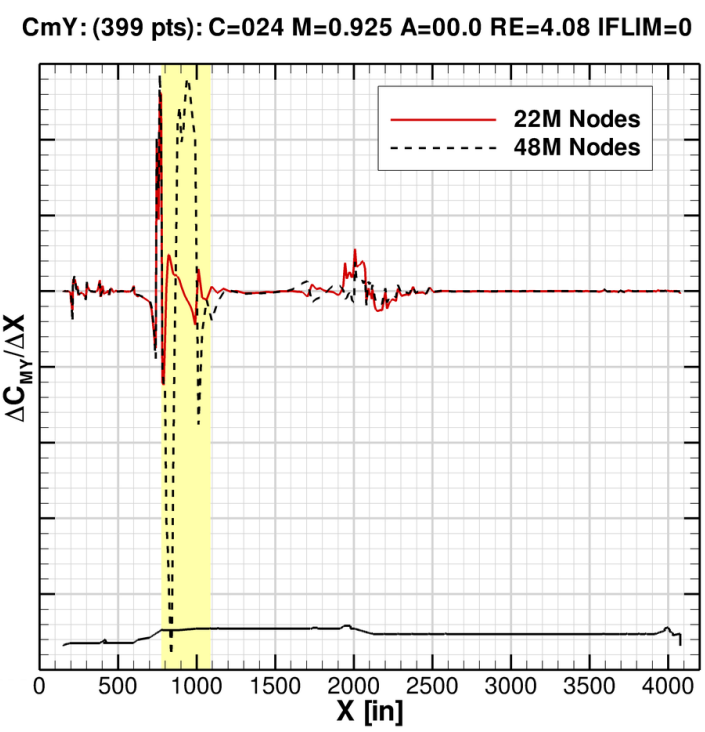

(a) $\mathrm{M}=0.925$
CmY: (399 pts): C=025 M=0.935 A=00.0 RE=4.08 IFLIM=0

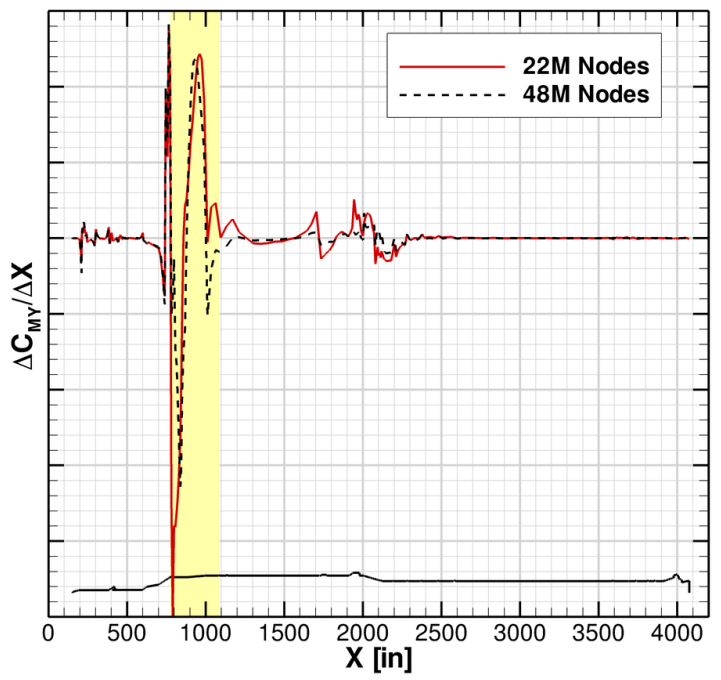

(b) $\mathrm{M}=0.935$

Figure 12. $\alpha=0^{\circ}$ : Pitching moment line loads for baseline and fine grids.

CmZ: (399 pts): $C=024$ M=0.925 A=00.0 RE=4.08 IFLIM=0

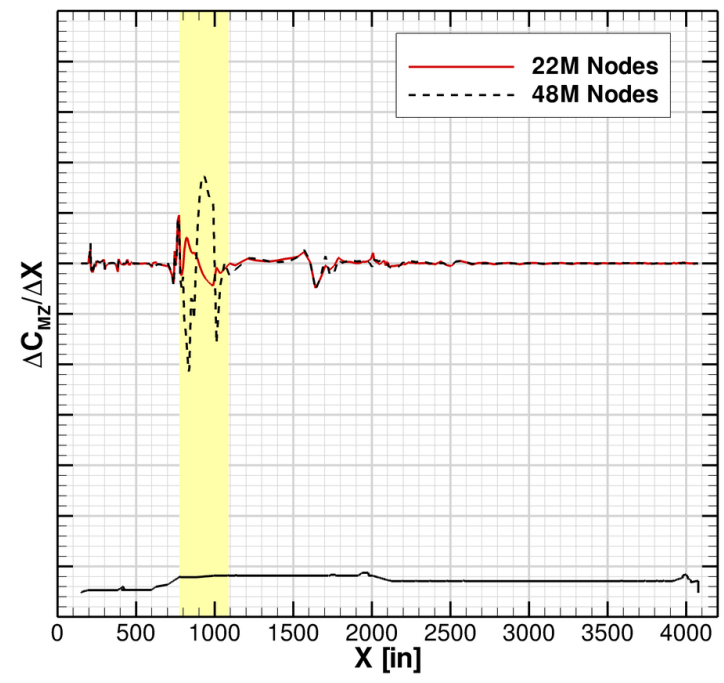

(a) $\mathrm{M}=0.925$
CmZ: (399 pts): C=025 M=0.935 A=00.0 RE=4.08 IFLIM=0

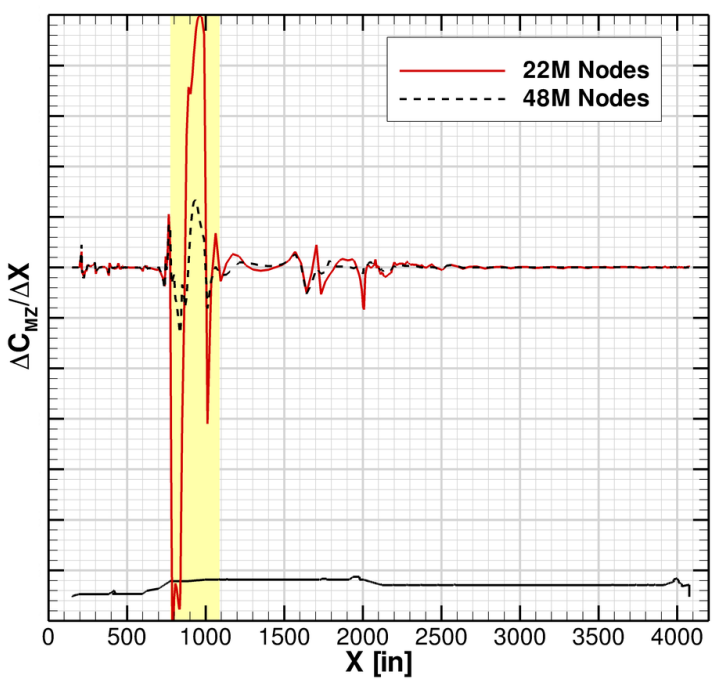

(b) $\mathrm{M}=0.935$

Figure 13. $\alpha=0^{\circ}$ : Yaw moment line loads for baseline and fine grids. 


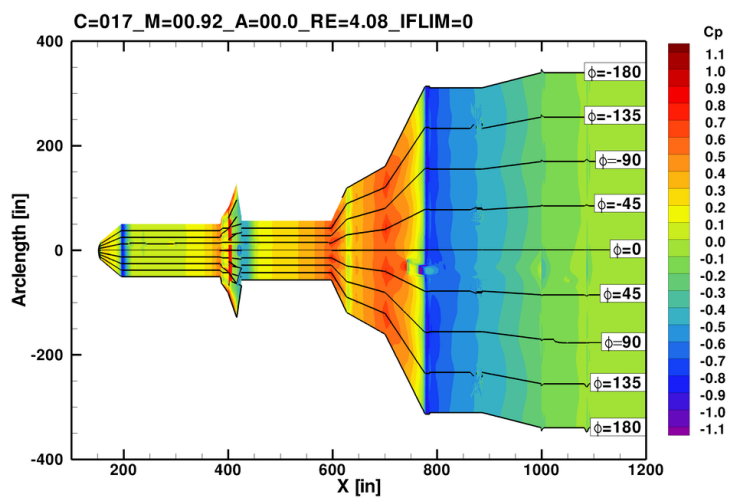

(a) $\mathrm{M}=0.920: 22$ million nodes

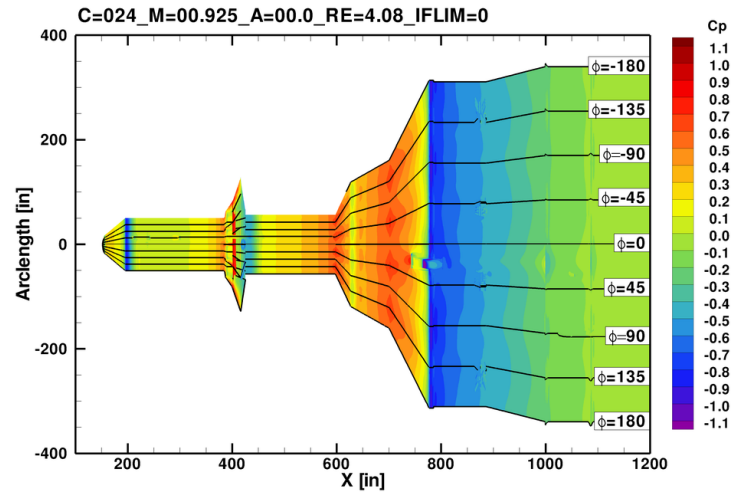

(c) $\mathrm{M}=0.925: 22$ million nodes

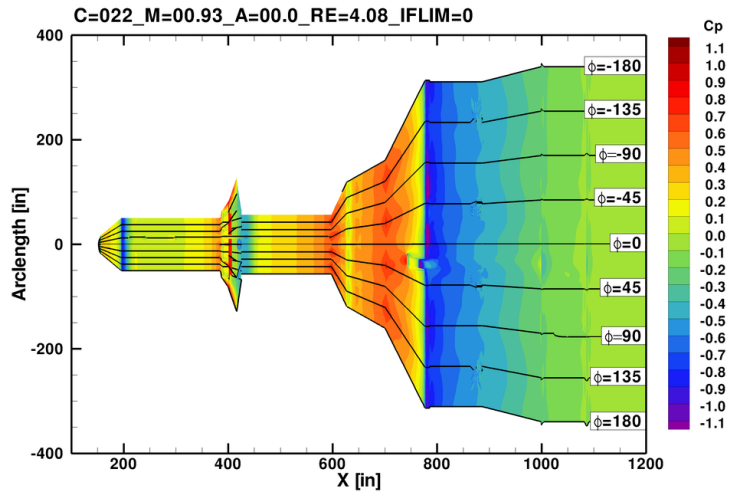

(e) $\mathrm{M}=0.930$ : 22 million nodes

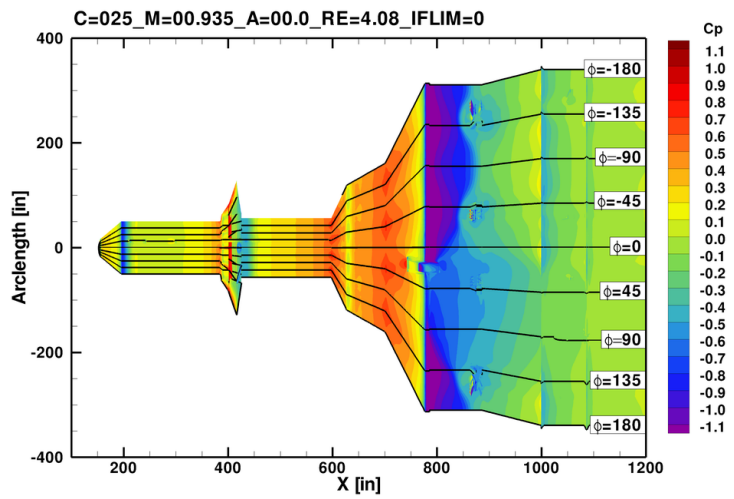

(g) $\mathrm{M}=0.935: 22$ million nodes

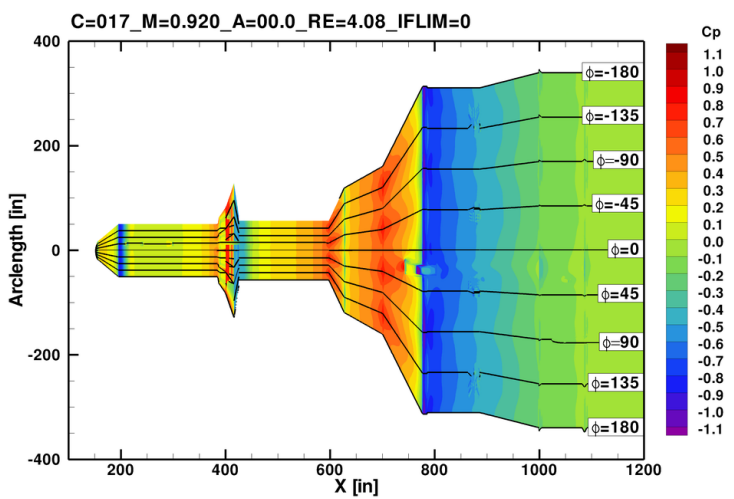

(b) $\mathrm{M}=0.920: 48$ million nodes

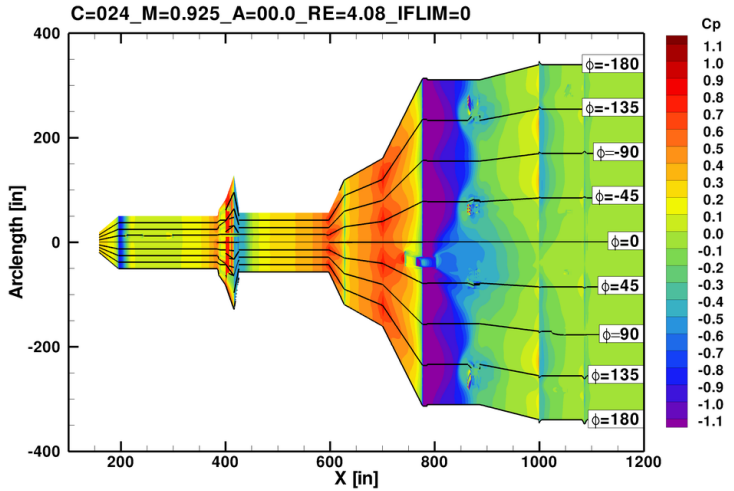

(d) $\mathrm{M}=0.925: 48$ million nodes

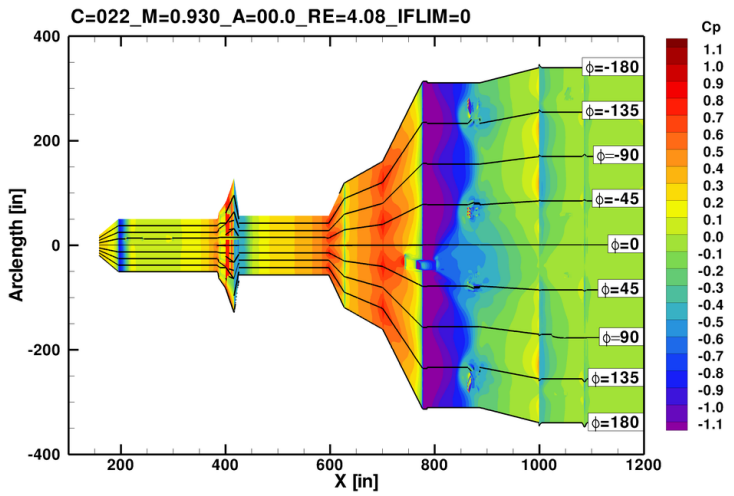

(f) $\mathrm{M}=0.930$ : 48 million nodes

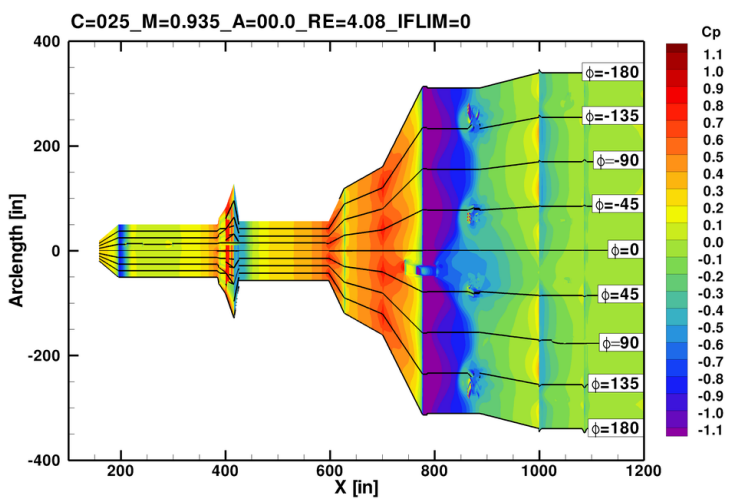

(h) $\mathrm{M}=0.935: 48$ million nodes

Figure 14. $\alpha=0^{\circ}: C_{p}$ contours on unrolled surface for baseline and fine grids. 


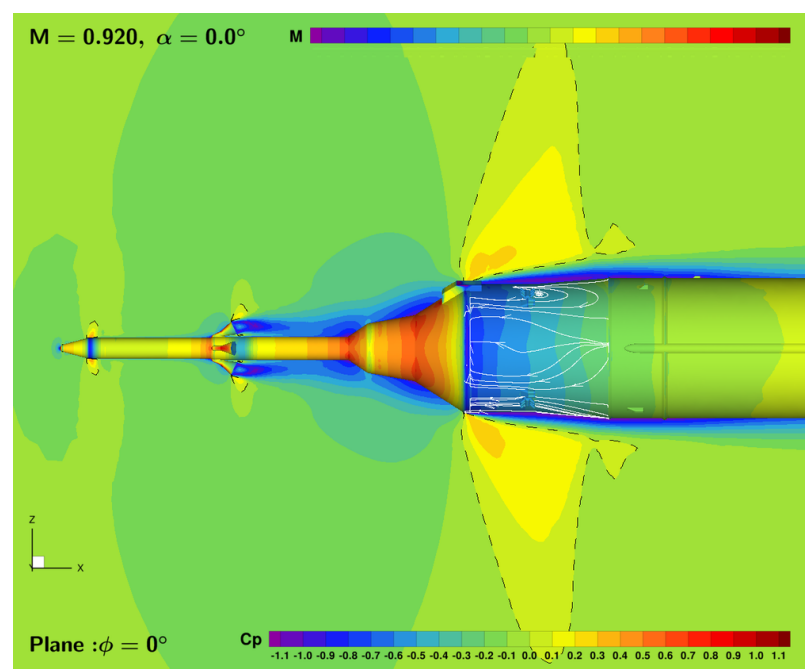

(a) $\mathrm{M}=$ 0.920: 22 million nodes

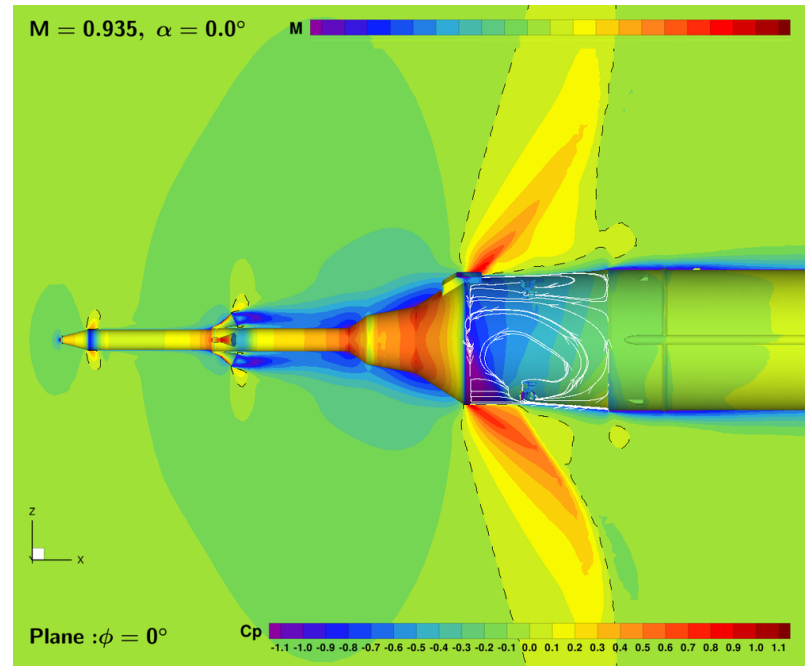

(c) $\mathrm{M}=0.935: 22$ million nodes

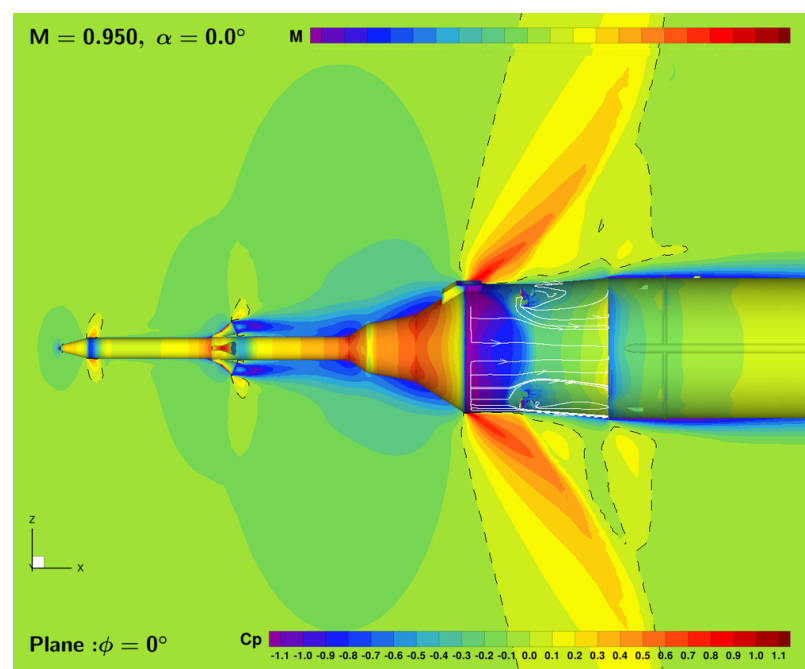

(e) $\mathrm{M}=0.950: 22$ million nodes

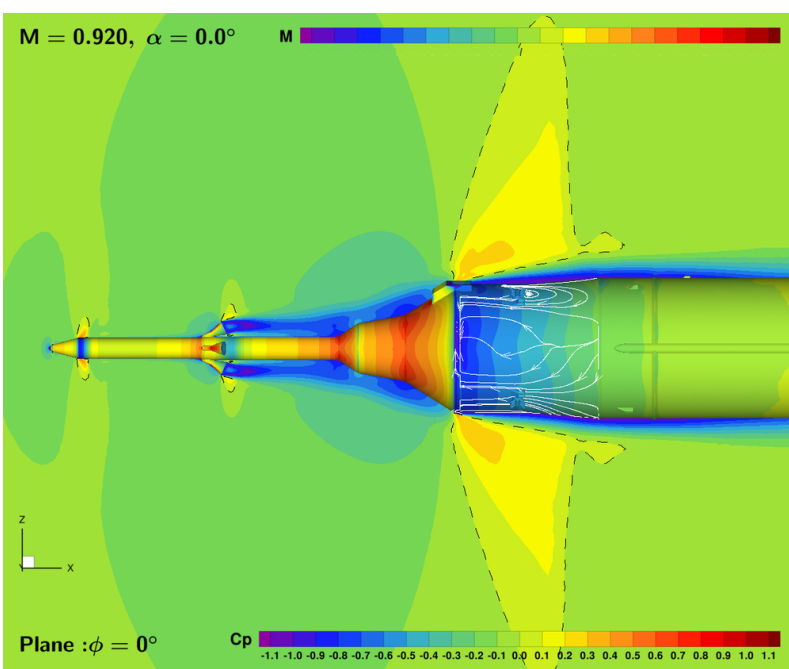

(b) $\mathrm{M}=$ 0.920: 48 million nodes

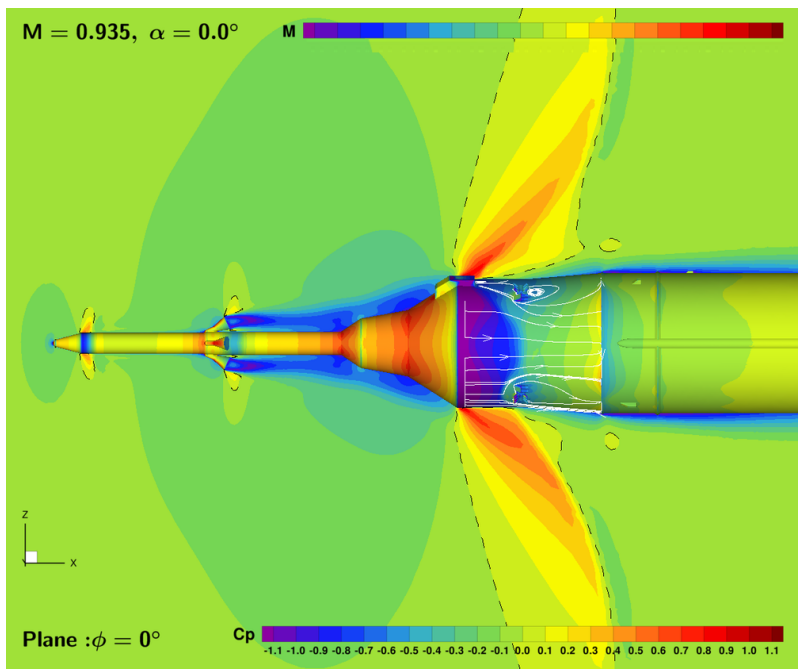

(d) $\mathrm{M}=0.935$ : 48 million nodes

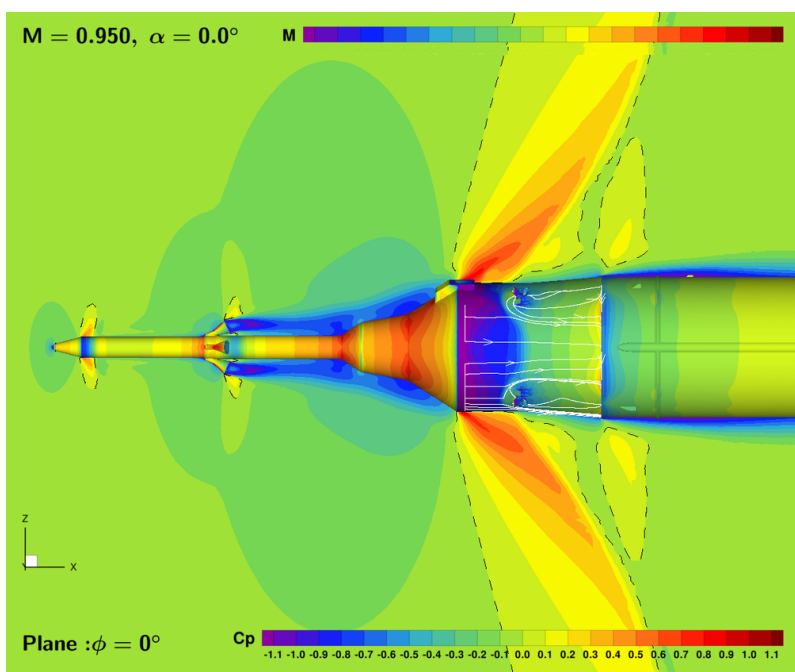

(f) $\mathrm{M}=0.950: 48$ million nodes

Figure 15. $\alpha=0^{\circ}$ : Mach number and surface $C_{p}$ contours with surface streamlines on the service module for baseline and fine grids. Sonic line shown dashed. 


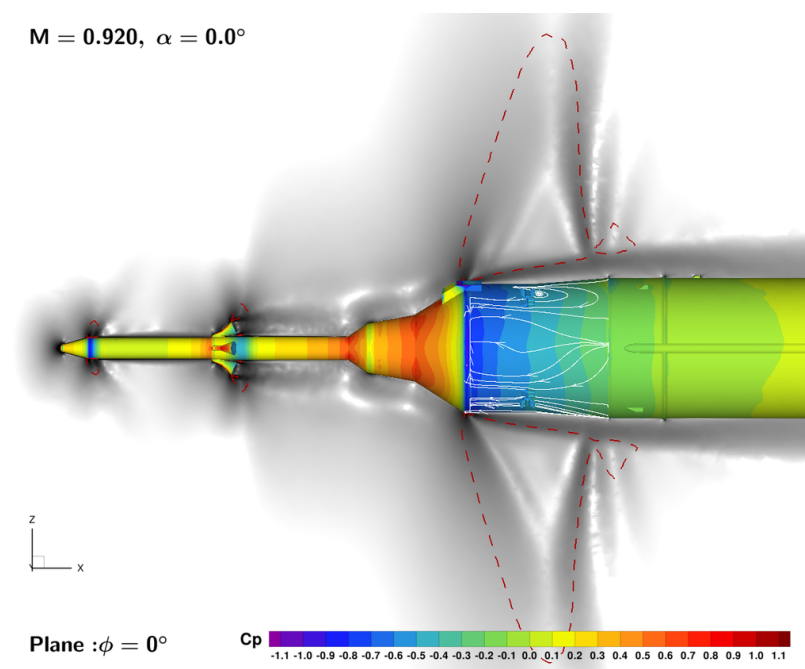

(a) $\mathrm{M}=0.920$ : 22 million nodes

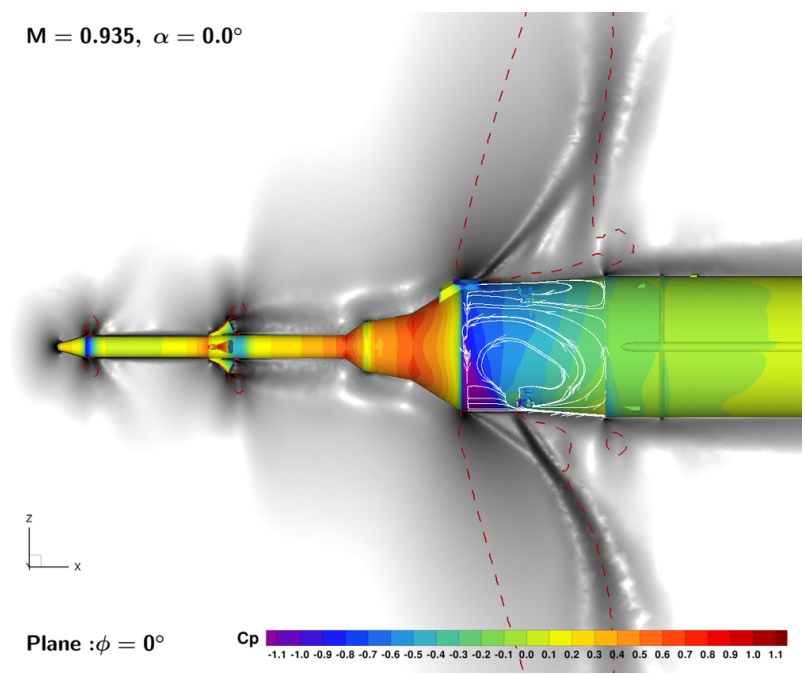

(c) $\mathrm{M}=0.935: 22$ million nodes

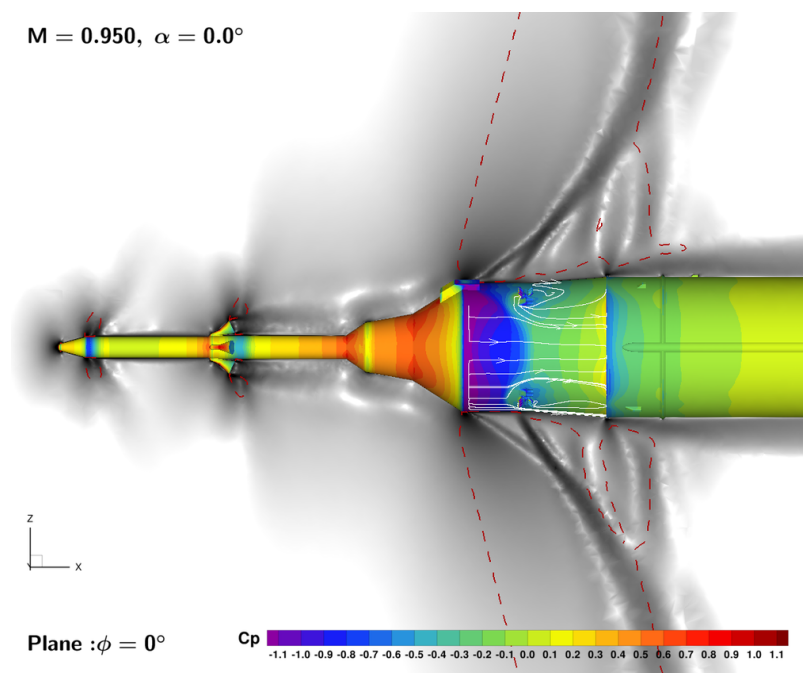

(e) $\mathrm{M}=0.950: 22$ million nodes

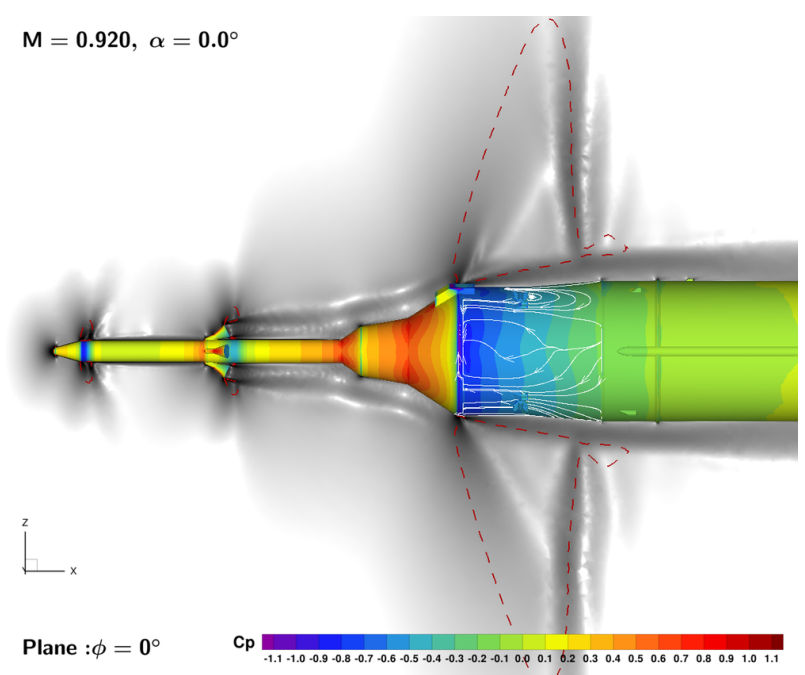

(b) $\mathrm{M}=0.920: 48$ million nodes

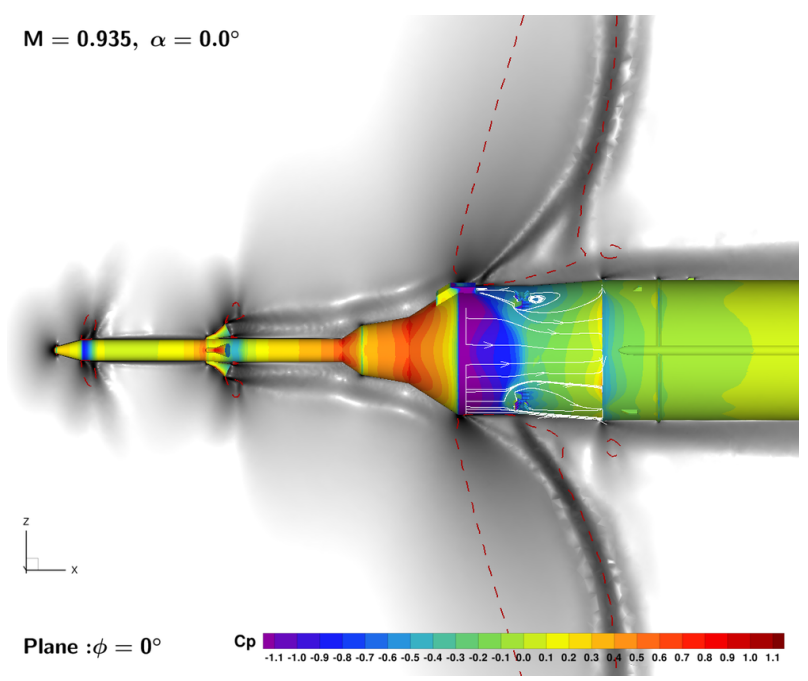

(d) $\mathrm{M}=0.935$ : 48 million nodes

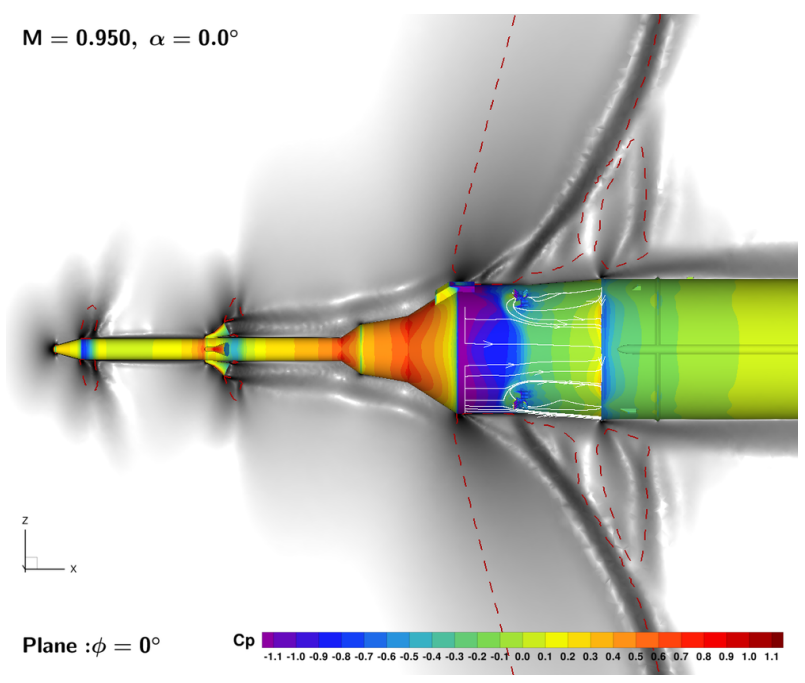

(f) $\mathrm{M}=$ 0.950: 48 million nodes

Figure 16. $\alpha=0^{\circ}$ : Simulated schlieren and surface $C_{p}$ contours with surface streamlines on the service module for baseline and fine grids. Sonic line shown dashed in red. 

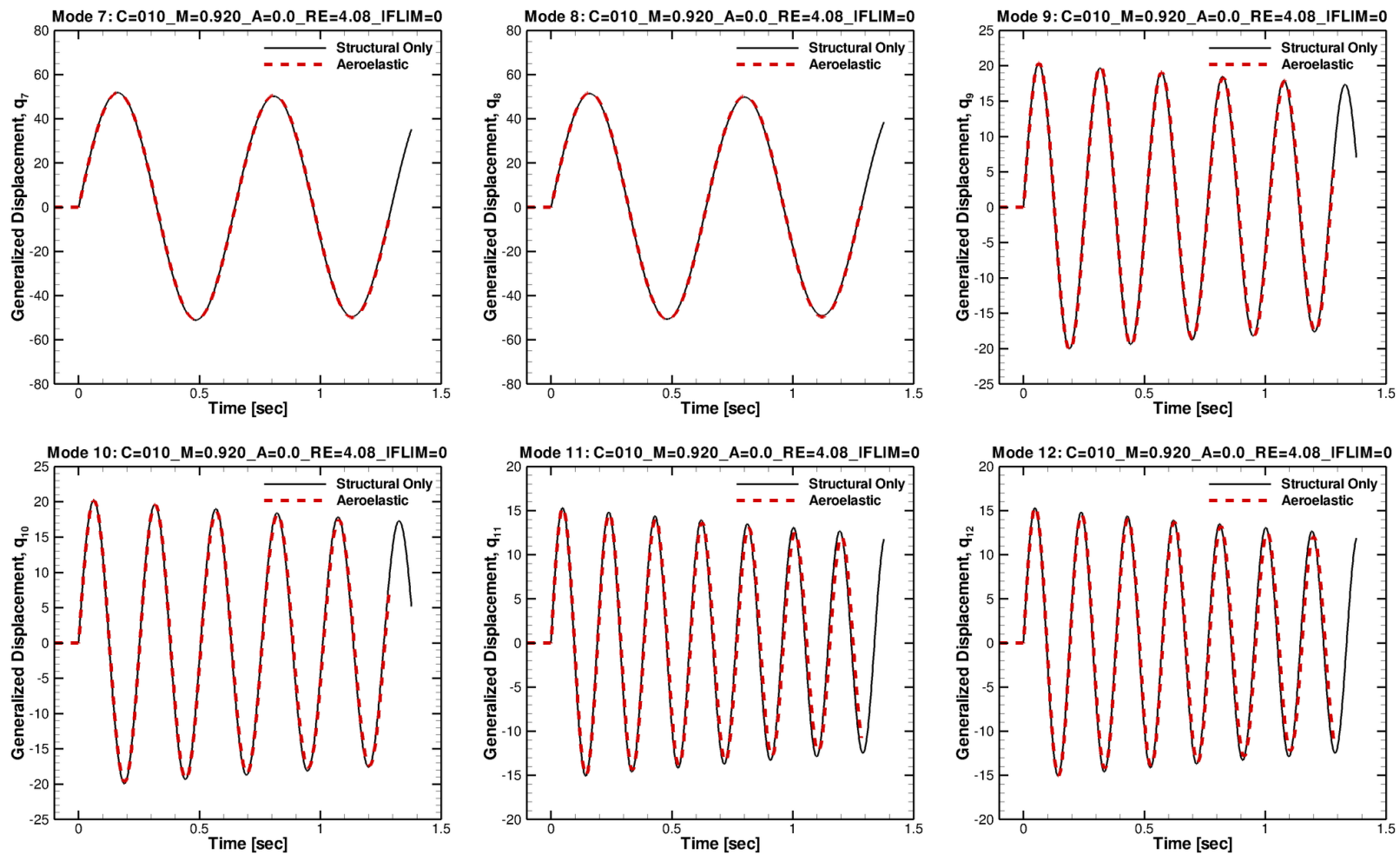

Figure 17. Fine grid, $\alpha=0^{\circ}, M=0.920$ : Generalized displacement history of first six elastic modes in response to a large initial generalized velocity perturbation.

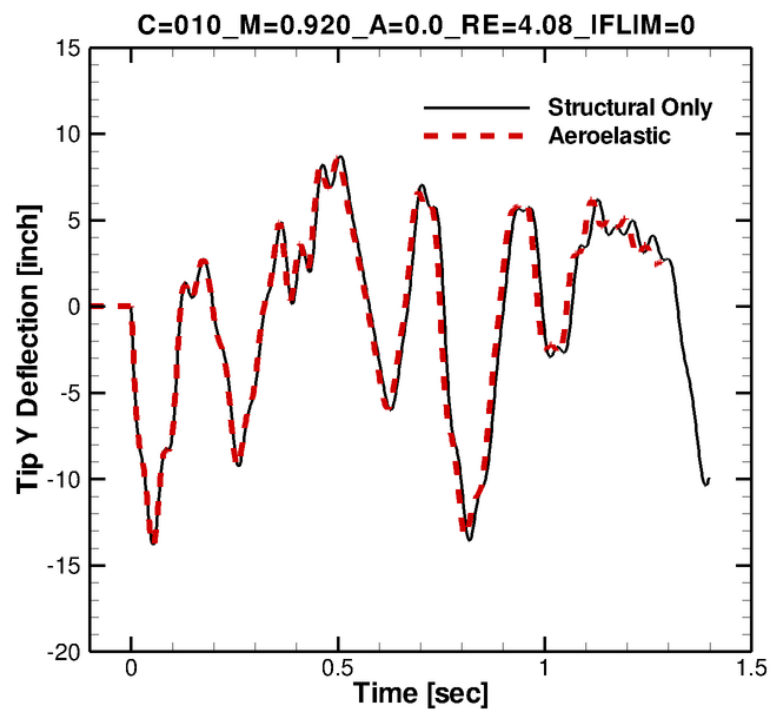

(a) Side (Y) component

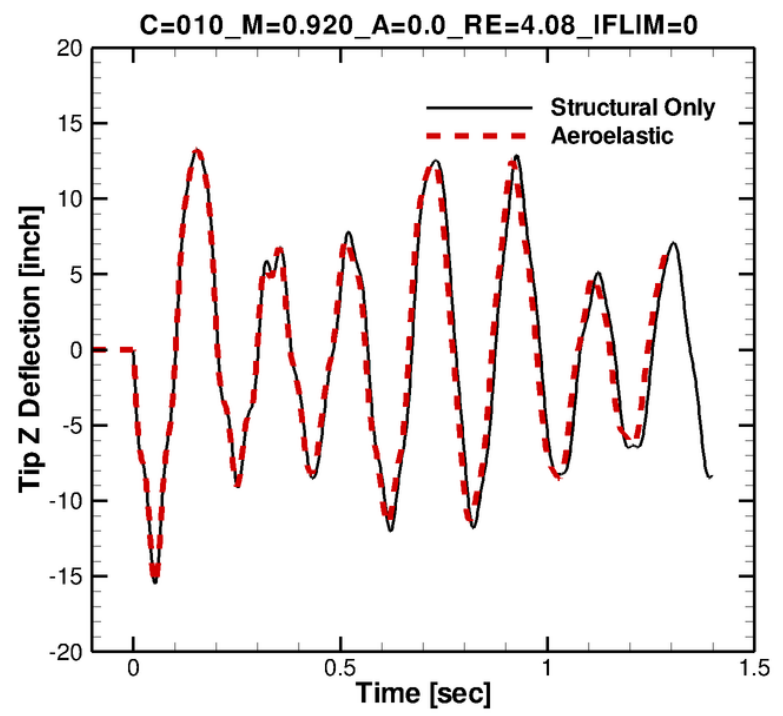

(b) Normal (Z) component

Figure 18. Fine grid, $\alpha=0^{\circ}, M=0.920$ : Tip displacement history for large initial generalized velocity perturbation. 


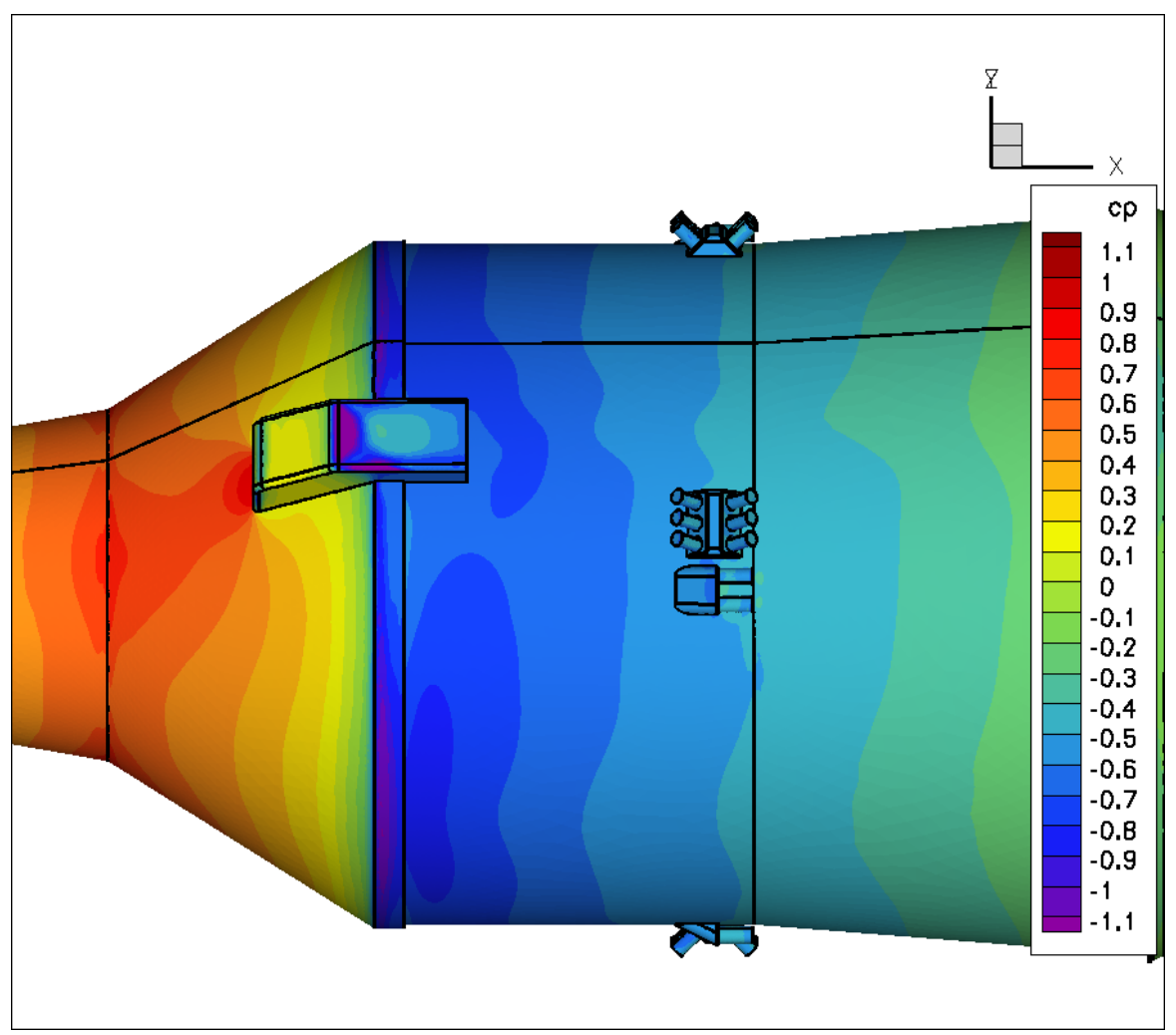

(a) Side

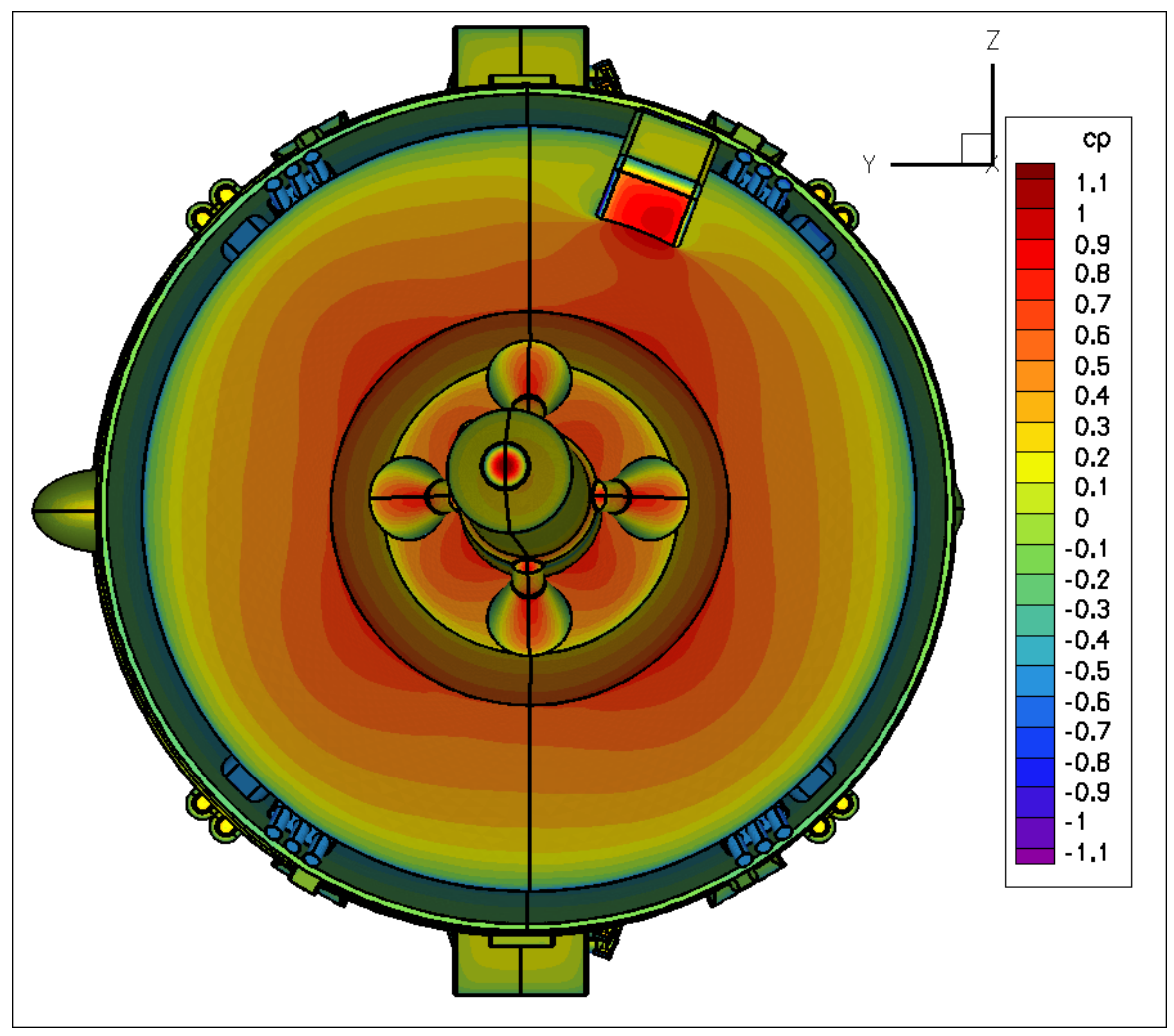

(b) Front

Figure 19. Fine grid, $\alpha=0^{\circ}, \mathrm{M}=0.920$ : Snapshot of $C_{p}$ contours on deflected surface. 

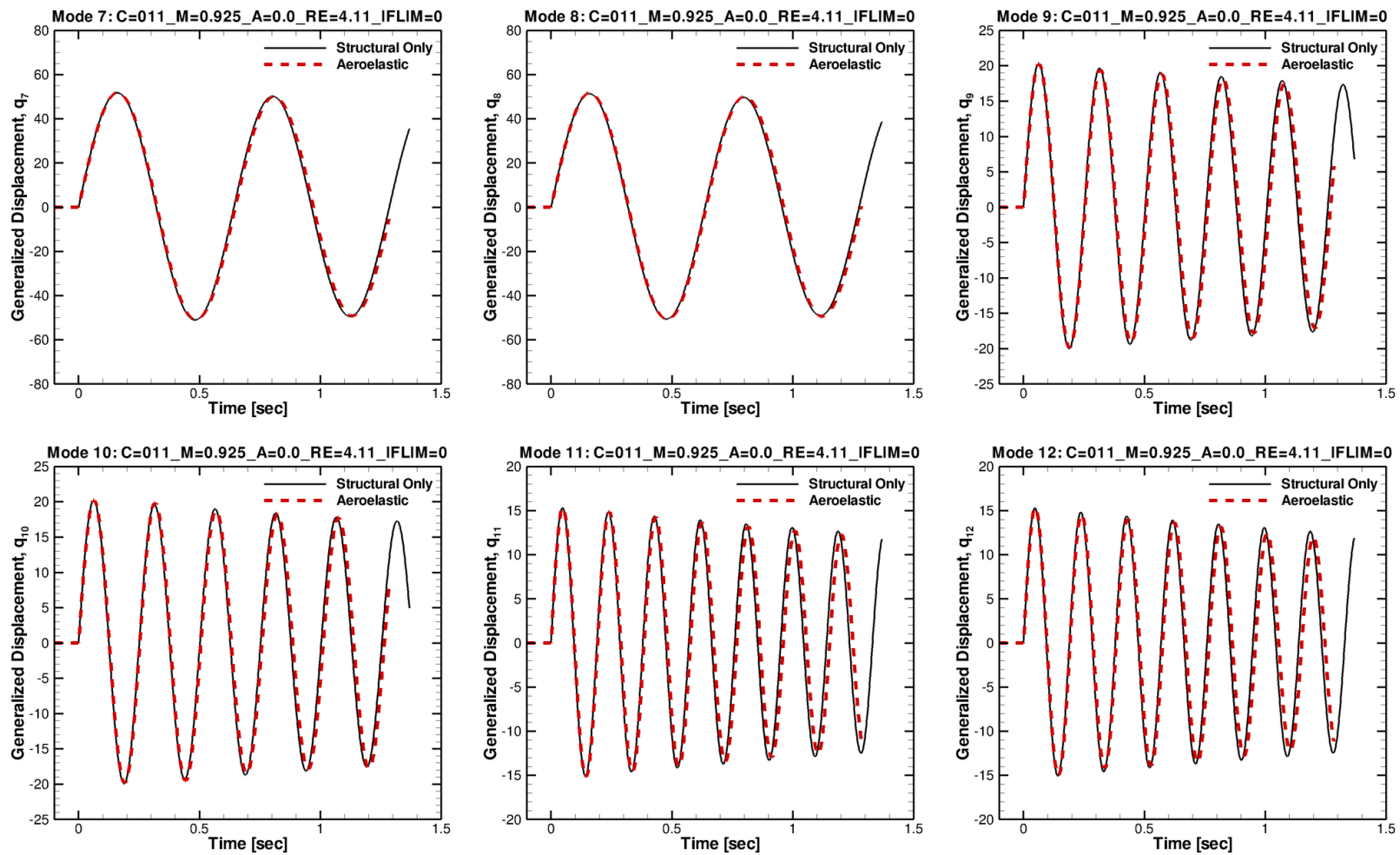

Figure 20. Fine grid, $\alpha=0^{\circ}, M=0.925$ : Generalized displacement history of first six elastic modes in response to a large initial generalized velocity perturbation.

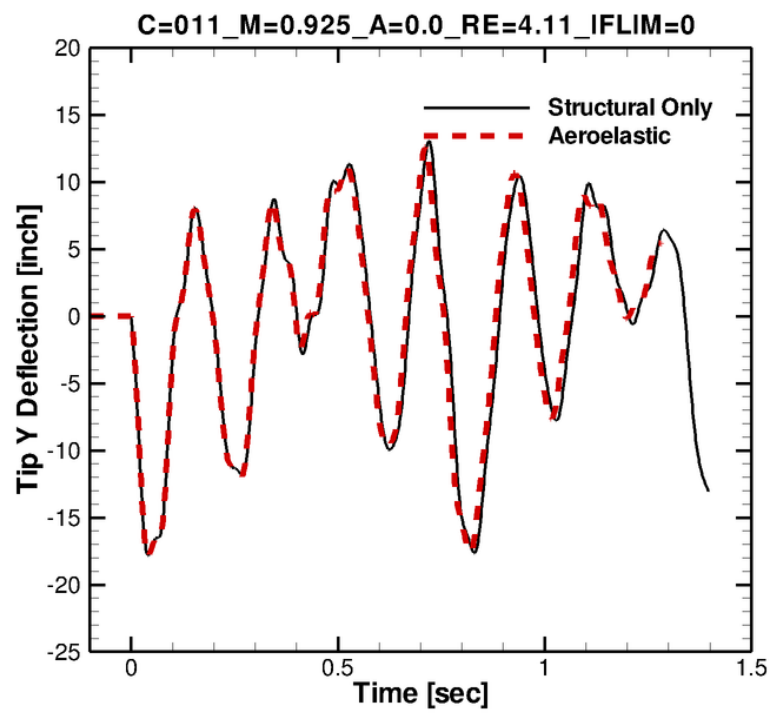

(a) Side (Y) component

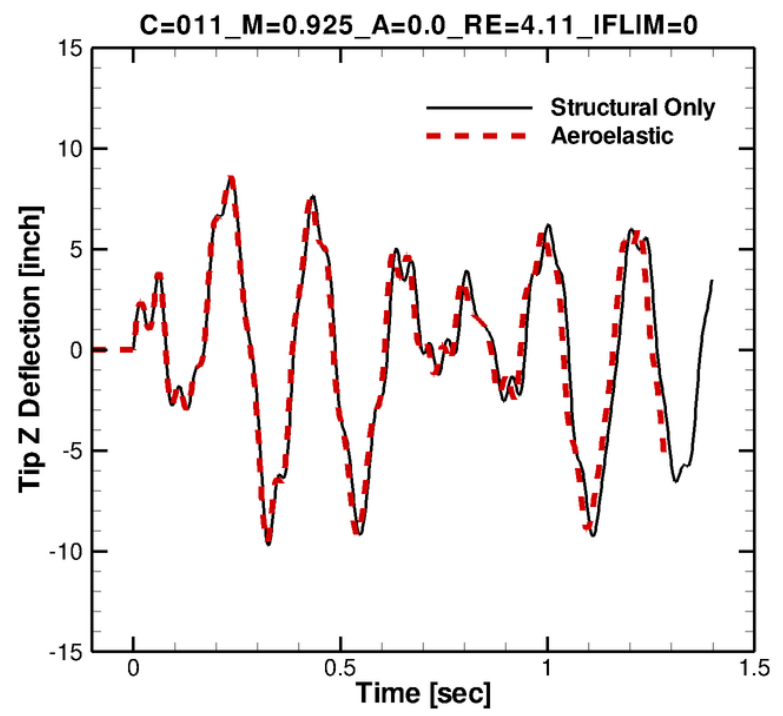

(b) Normal (Z) component

Figure 21. Fine grid, $\alpha=0^{\circ}, M=0.925$ : Tip displacement history for large initial generalized velocity perturbation. 


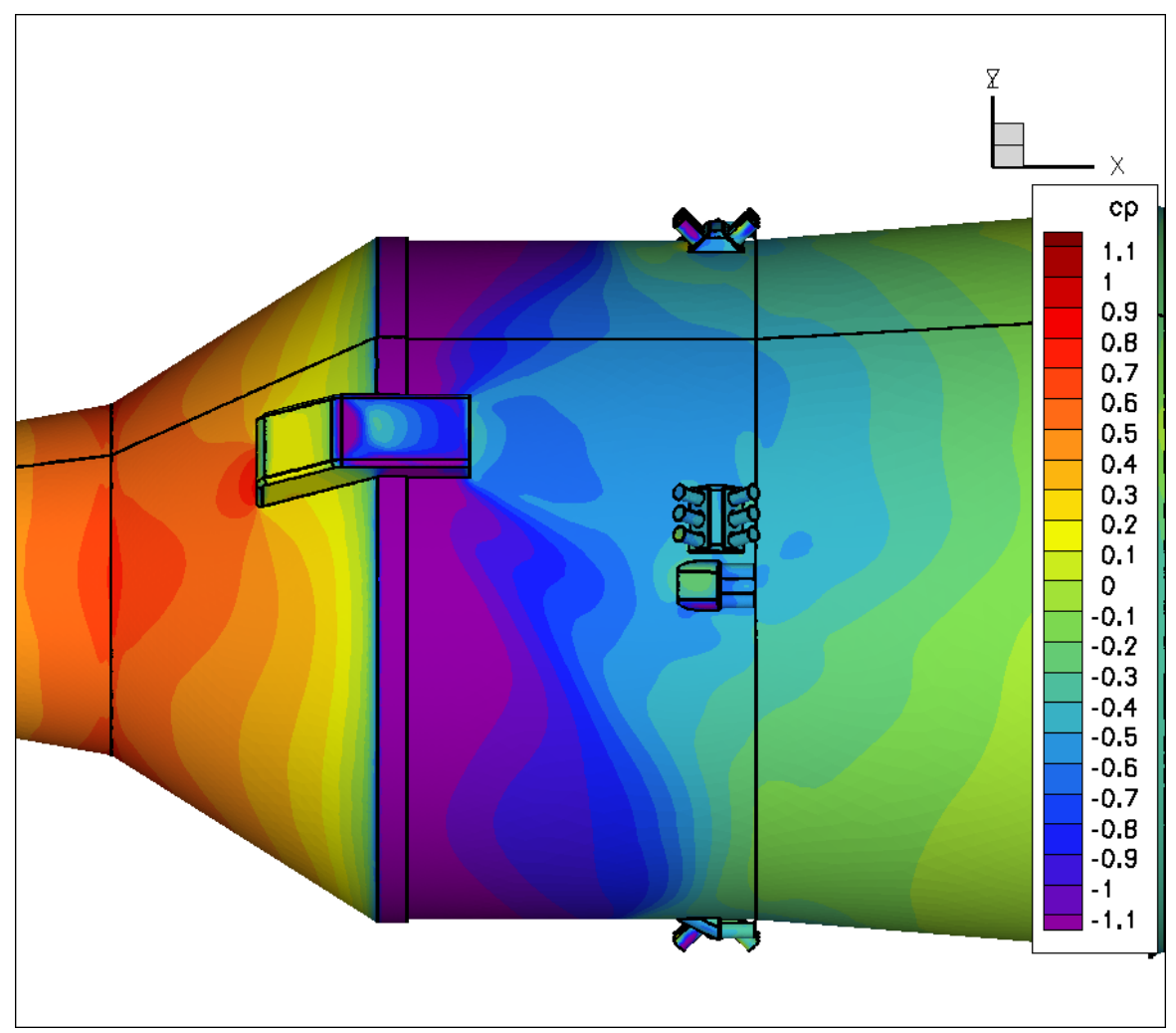

(a) Side

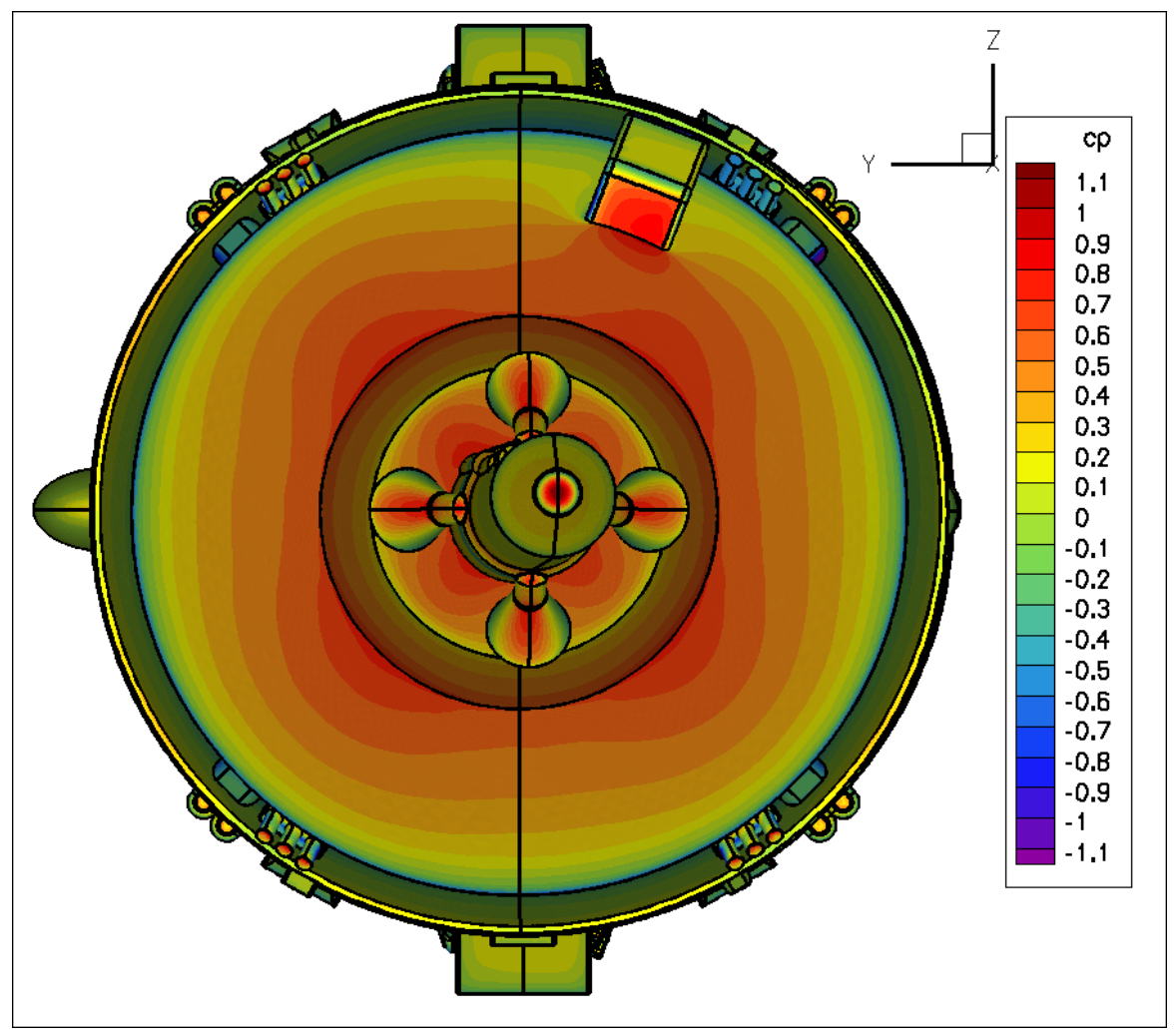

(b) Front

Figure 22. Fine grid, $\alpha=0^{\circ}, \mathrm{M}=0.925$ : Snapshot of $C_{p}$ contours on deflected surface. 


\section{Side and Normal Force vs Mach}

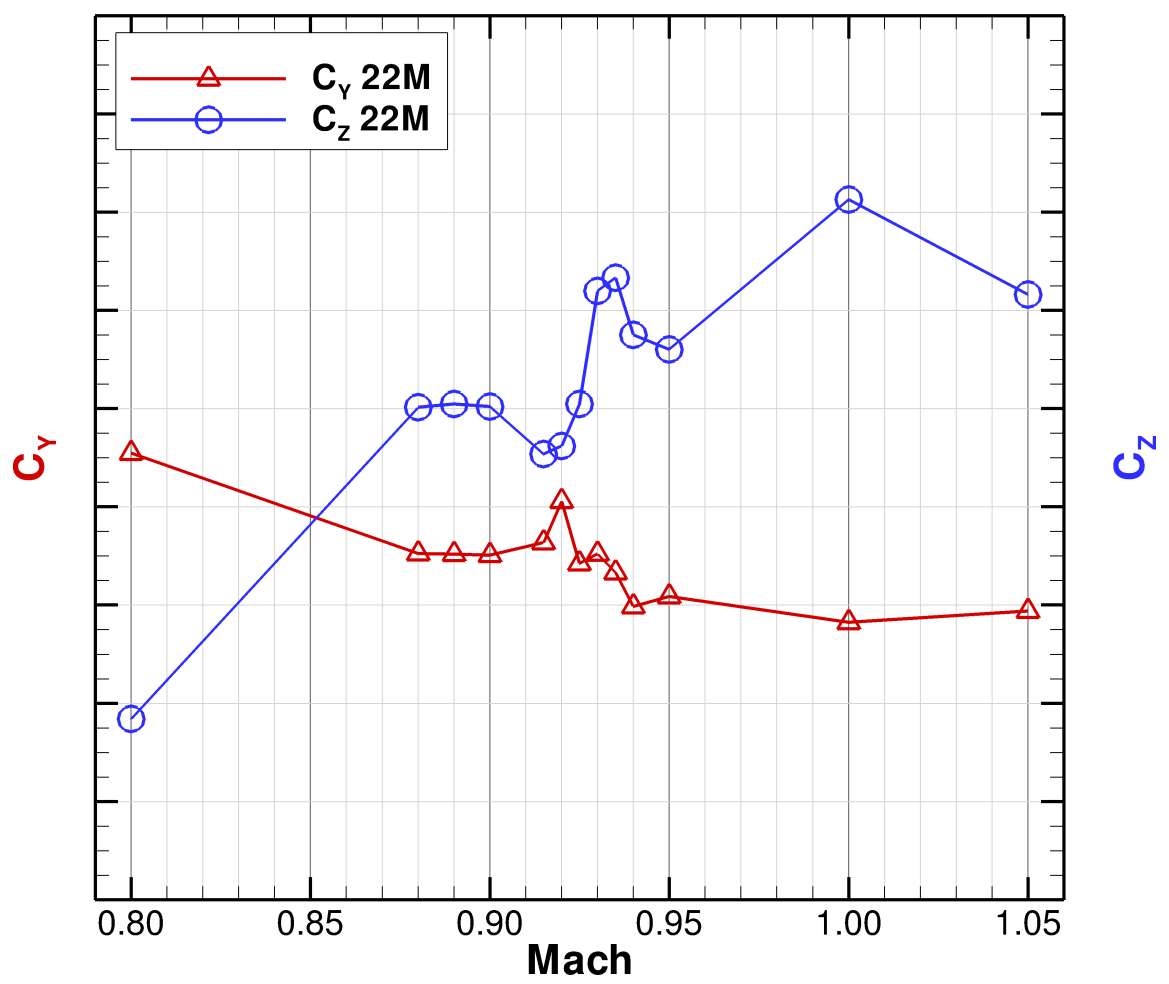

(a) Side $\left(C_{Y}\right)$ and normal $\left(C_{Z}\right)$ forces.

Pitch and Yaw Moments vs Mach

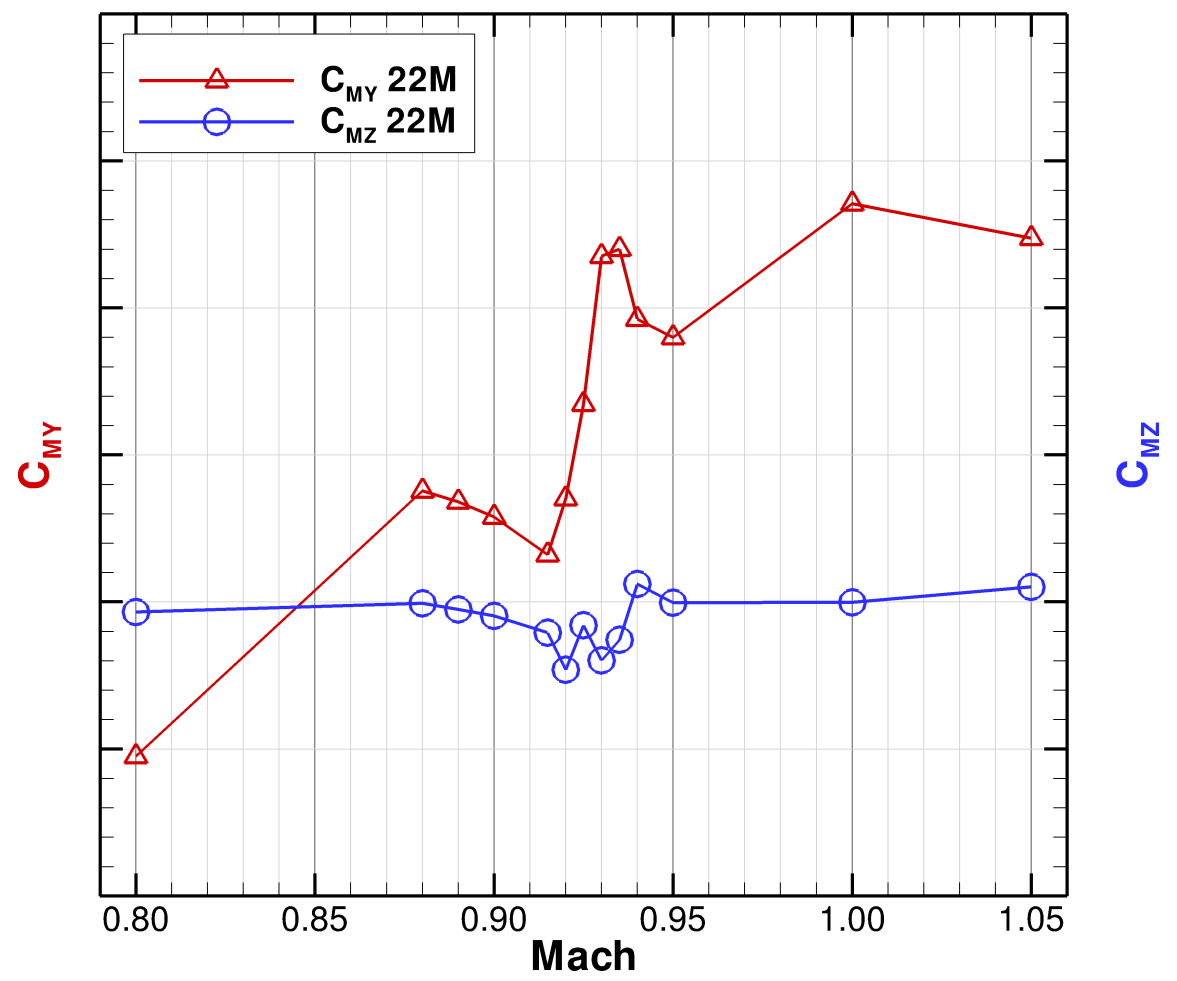

(b) Pitching $\left(C_{M Y}\right)$ and yawing $\left(C_{M Z}\right)$ moments.

Figure 23. Baseline 22 million node grid, $\alpha=2.5^{\circ}$ : Integrated forces and moments. 


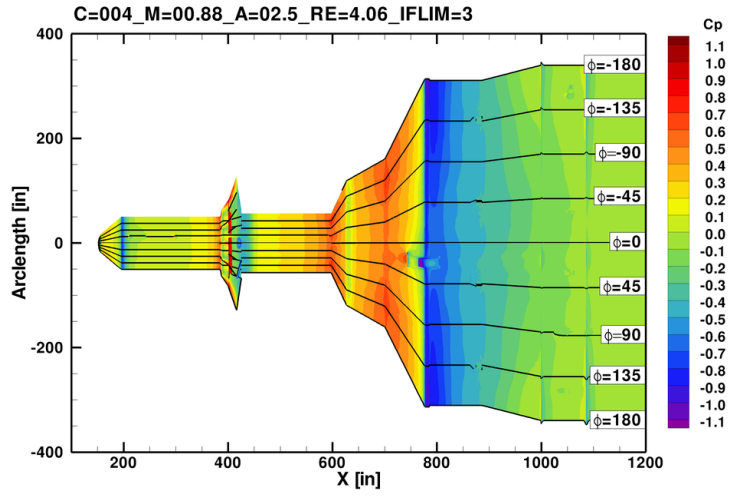

(a) $\mathrm{M}=0.880$

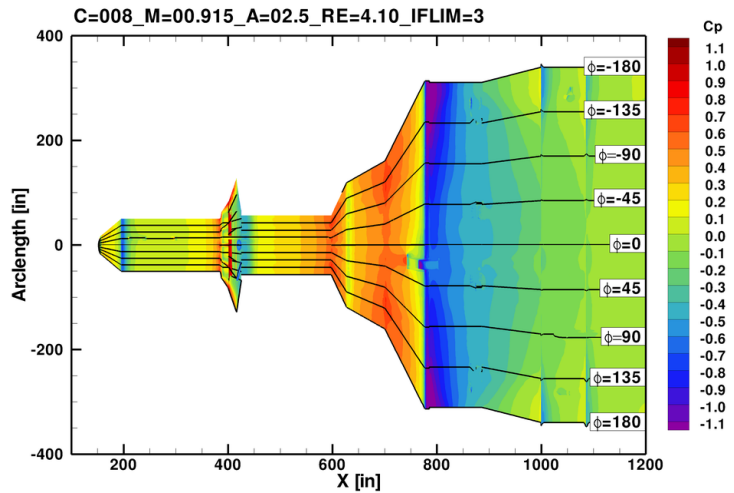

(c) $\mathrm{M}=0.915$

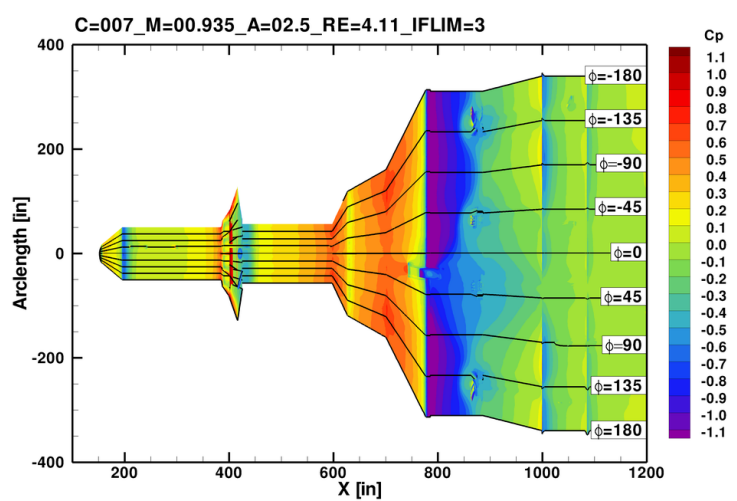

(e) $\mathrm{M}=0.935$

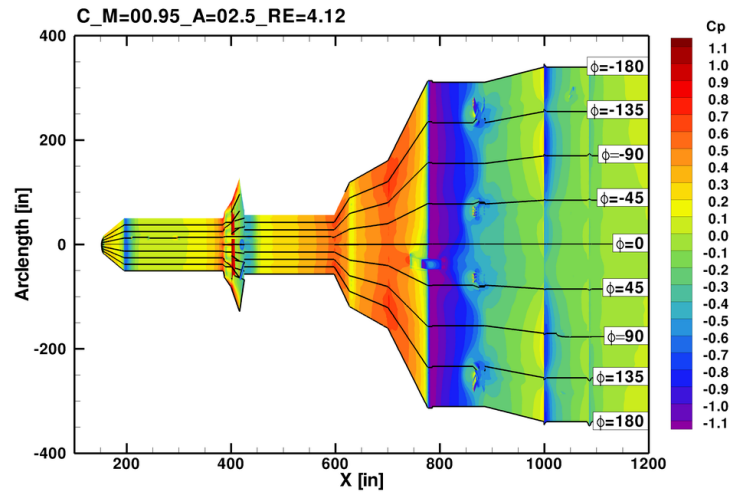

(g) $M=0.950$

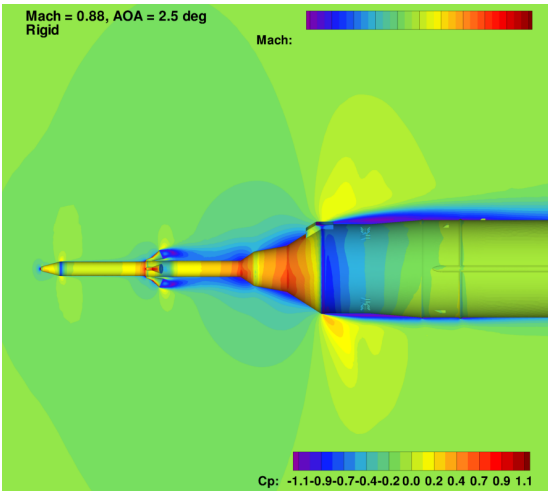

(b) $\mathrm{M}=0.880$

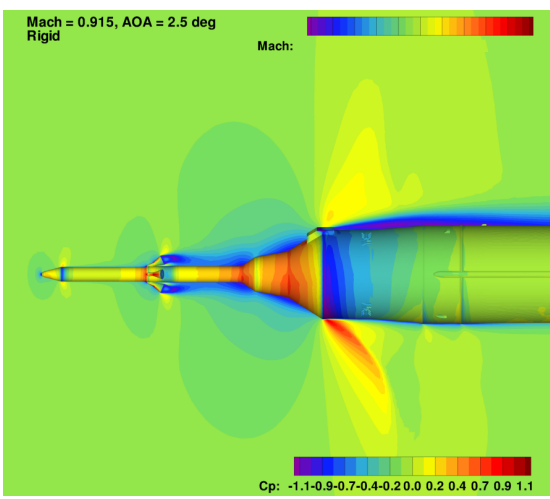

(d) $\mathrm{M}=0.915$

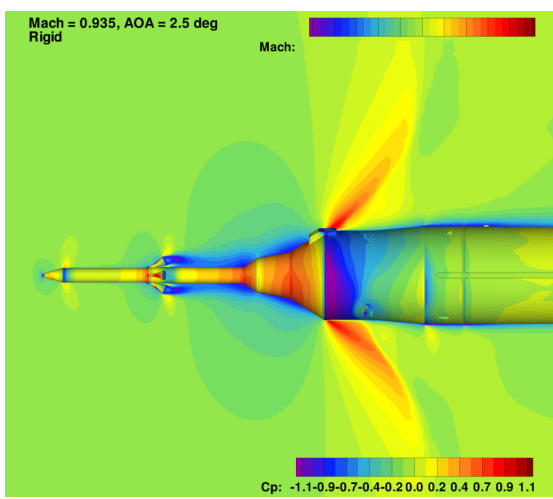

(f) $\mathrm{M}=0.935$

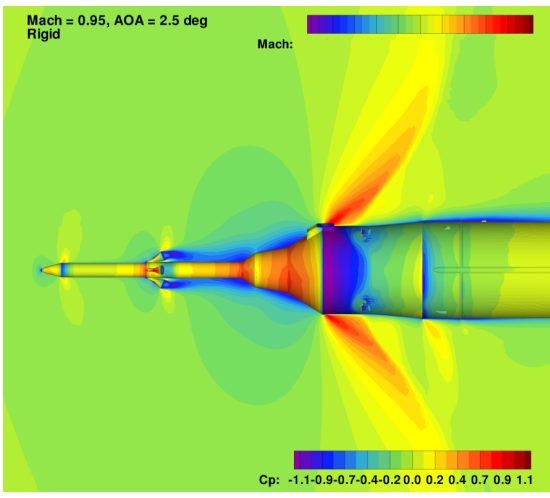

(h) $\mathrm{M}=0.950$

Figure 24. Baseline grid, $\alpha=2.5^{\circ}: C_{p}$ contours on unrolled surface (left). Mach number contours on $\phi=0^{\circ}$ plane and $C_{p}$ contours on surface (right). 